ARTICLE

https://doi.org/10.1038/s41467-019-12197-3

\title{
Maladaptive activation of Nav1.9 channels by nitric oxide causes triptan-induced medication overuse headache
}

Caroline Bonnet ${ }^{1}$, Jizhe Hao ${ }^{1}$, Nancy Osorio ${ }^{1}$, Anne Donnet ${ }^{2}$, Virginie Penalba ${ }^{1}$, Jérôme Ruel ${ }^{1}$ \& Patrick Delmas ${ }^{1}$

Medication-overuse headaches $(\mathrm{MOH})$ occur with both over-the-counter and pain-relief medicines, including paracetamol, opioids and combination analgesics. The mechanisms that lead to $\mathrm{MOH}$ are still uncertain. Here, we show that abnormal activation of Nav1.9 channels by Nitric Oxide (NO) is responsible for $\mathrm{MOH}$ induced by triptan migraine medicine. Deletion of the Scn11a gene in $\mathrm{MOH}$ mice abrogates $\mathrm{NO}$-mediated symptoms, including cephalic and extracephalic allodynia, photophobia and phonophobia. NO strongly activates Nav1.9 in dural afferent neurons from $\mathrm{MOH}$ but not normal mice. Abnormal activation of Nav1.9 triggers CGRP secretion, causing artery dilatation and degranulation of mast cells. In turn, released mast cell mediators potentiates Nav1.9 in meningeal nociceptors, exacerbating inflammation and pain signal. Analysis of signaling networks indicates that PKA is downregulated in trigeminal neurons from $\mathrm{MOH}$ mice, relieving its inhibitory action on NO-Nav1.9 coupling. Thus, anomalous activation of Nav1.9 channels by NO, as a result of chronic medication, promotes $\mathrm{MOH}$.

\footnotetext{
${ }^{1}$ Aix-Marseille-Université, CNRS, Laboratoire de Neurosciences Cognitives, UMR 7291, CS8011, Bd Pierre Dramard, 13344 Marseille, France. ${ }^{2}$ Centre d'évaluation et de traitement de la douleur, Hôpital de la Timone, Marseille, France. Correspondence and requests for materials should be addressed to P.D. (email: patrick.delmas@univ-amu.fr)
} 
C hronic headache encompasses many different headache diagnoses and include chronic migraines, chronic tensiontype headaches, medication-overuse headaches $(\mathrm{MOH})$, and other types of daily persistent headaches. Migraine, a frequently incapacitating neurovascular disorder, affects hundreds of millions of individuals worldwide ${ }^{1}$. It is characterized by a severe, debilitating and throbbing unilateral headache accompanied by a host of neurological symptoms including hypersensitivity to visual and auditory stimulation, nausea and vomiting, and a variety of autonomic, cognitive and motor disturbances ${ }^{2,3}$.

Current antimigraine drugs target trigeminovascular $5-\mathrm{HT}_{1 \mathrm{~B}}$ / ${ }_{1 \mathrm{D}}, 5-\mathrm{HT}_{1 \mathrm{~F}}$, and CGRP receptors. These different antimigraine medications induce adverse side effects, including $\mathrm{MOH}$, which is a worldwide health problem with a prevalence range of $1-2 \%$ with a 3:1 female to male ratio. $\mathrm{MOH}$ is a severe form of headache where the patients are prone to develop primary headaches with unsuccessful treatments. Patients with migraine or tension-type headaches have a higher potential for developing $\mathrm{MOH}$ than other primary headaches. $\mathrm{MOH}$ does not develop in persons without a history of headache when medication is being used for other conditions, such as inflammatory diseases. In addition, virtually all medication for headaches may lead to $\mathrm{MOH}$, including opioids, ergotamine, butalbital-containing medicine, triptans or a combination thereof. Thus, $\mathrm{MOH}$ is generated in headache-prone persons by the interaction between headache medication and pre-existing headache disorder pathways.

The precise mechanisms that lead to $\mathrm{MOH}$ development are largely unknown. However, multiple factors may be implicated, including genetic predisposition, sensitization within the trigeminal (TG) system, abnormal cortical pain processing and decreased antinociceptive activity of the supraspinal structures $^{4-7}$.

Multiple studies indicate that migraine medication induces sensitization of peripheral and central pain pathways. For instance, chronic use of opioids and triptans in animals has been shown to increase the level of calcitonin gene-related peptide (CGRP), which is involved in neurogenic inflammation and headache pain ${ }^{8,9}$. These animals develop a persistent hypersensitivity or latent sensitization to provocative triggers, such as environmental stress stimulus and the well-known human migraine trigger nitric oxide (NO). This latent sensitization persists long after discontinuation of drug administration and produce a state of generalized cutaneous allodynia that was detected in periorbital regions and hind paw.

To probe molecular mechanisms that lead to $\mathrm{MOH}$, we developed a $\mathrm{MOH}$ mouse model based on the sustained administration of sumatriptan, a 5-HT receptor agonist selective for 5$\mathrm{HT}_{1 \mathrm{D}}$ and $5-\mathrm{HT}_{1 \mathrm{~B}}$ subtypes. We applied the $\mathrm{MOH}$ model to transgenic mice for studying the role of the nociceptor-specific voltage-gated Nav1.9 channel in headache. The function of this channel in the TG pain pathway is still poorly understood ${ }^{10-12}$. Nav1.9 channel is known to generate a persistent, tetrodotoxin (TTX)-resistant $\mathrm{Na}^{+}$current that promotes sustained neuronal activity in dorsal root ganglion (DRG) neurons ${ }^{13-17}$. Nav1.9 is known to contribute to both inflammatory ${ }^{18-22}$ and neuropathic ${ }^{22,23}$ somatic pain in animal models, and variants of the Scn11a gene encoding Nav1.9 in humans lead to congenital insensitivity to pain and to painful syndromes ${ }^{24-27}$.

Using molecular, electrophysiological, and behavioral approaches, we show that mice chronically treated with sumatriptan display increased responsiveness of Nav1.9 to NO, leading to headache/migraine-like symptoms including generalized allodynia, photophobia, and phonophobia. $\mathrm{MOH}$ mice show deficit in PKA-mediated inhibition of NO-Nav1.9 coupling, causing hyperactivity of meningeal nociceptors and inflammation in the meninges. Thus, our data identify abnormal activation of Nav1.9 by $\mathrm{NO}$ as a central determinant of triptan-induced $\mathrm{MOH}$ and pave the way for the development of mechanism-based treatment strategies that can improve the management of primary headaches.

\section{Results}

Nav1.9 is expressed in meningeal nociceptors. Immunostaining of mouse TG cryosections indicated that Nav1.9 is expressed in $32 \%$ of TG neurons (Fig. 1a). Nearly all immunopositive neurons (96\%, $n=174)$ exhibited small-diameter or medium-diameter cell bodies $(<27 \mu \mathrm{m}$, average largest diameter $\varnothing=17.4 \pm 0.3 \mu \mathrm{m})$ (Fig. 1b), while a minority of them $(3.8 \%, n=7$ out of 181$)$ displayed large cell bodies $(\varnothing \geq 27 \mu \mathrm{m})$. Small peripherin-positive fibers showed features of apposition with meningeal arteries in whole mount of mouse dura mater (Supplementary Fig. 1A). Dual-labeling showed that $89 \%(n=124 / 139)$ and $91 \%(n=101)$ of peripherin-positive meningeal fibers were immunoreactive for Nav1.9 and CGRP, respectively (Fig. 1c, d), suggesting that Nav1.9 and CGRP co-distribute in a large proportion of meningeal fibers. Double-labeling for Nav1.9 and CGRP could not be made due to the different fixation conditions of primary antibodies. However, the quasi-totality of $\beta$-gal -positive TG neurons exhibited CGRP staining in cryosections from Scn11a-GAL reporter transgenic mice $(n=63 / 64)$ (Supplementary Fig. 1B). Few Nav1.9-positive meningeal fibers were also found to be immunoreactive for NF200, possibly corresponding to some lightly myelinated sensory fibers (Supplementary Fig. 1C, D). A majority (57\%) of retrogradely labeled (DiI) dural afferent neurons was found to express Nav1.9 current using patch clamp recording (Fig. 1e, g). Consistently, $54 \%$ of $\mathrm{DiI}^{+}$-dural afferent neurons from Scn11a-GAL reporter transgenic mice exhibited $\beta$ gal enzymatic activity (Fig. 1f, g). Together, these data provide evidence that about half of dural afferent neurons expresses functional Nav1.9 channels.

Scn11a gene inactivation abrogates NO-induced allodynia in MOH mice. To probe the role of Nav1.9 channels in $\mathrm{MOH}$, we developed a model of $\mathrm{MOH}$ in Nav1.9-1- mice and their wildtype (WT) littermates using triptan medicines and assessed quantitatively behavioral correlates of headache and migrainous symptoms, including generalized allodynia, photophobia, and phonophobia. Osmotic minipump infusion of sumatriptan in WT mice for 6 days $(0.6 \mathrm{mg} / \mathrm{kg} /$ day $)$ produced a timedependent reduction in mechanical withdrawal thresholds of the hind paw relative to saline-treated WT mice (Fig. 2a). Recovery of paw sensory thresholds to sumatriptan preinfusion values occurred within 18-20 days after minipump implantation (Fig. 2a). Latent sensitization to the migraine trigger NO was tested at day 21, once sensory thresholds were returned to pre-sumatriptan baseline levels (Fig. 2b). Injection of the NO donor sodium nitroprusside (SNP, $0.03 \mathrm{mg} / \mathrm{kg}$ ) into the loose skin over the neck evoked strong extracephalic tactile allodynia in sumatriptan-treated WT mice compared with saline-treated WT mice (Fig. 2b). Heightened SNP-induced allodynia in sumatriptan-treated WT mice was still observable up to 46 days after minipump implantation (Supplementary Fig. 2A). Heightened mechanical allodynia induced by SNP injection at day 21 was absent in sumatriptan-treated Nav1.9-I- mice (Fig. 2d). Moreover, saline-treated Nav1.9-I- and WT mice displayed similar SNP-induced (basal) allodynia (Fig. 2b, d), indicating that Nav1.9 contributes to the SNP-induced heightened allodynia in sumatriptan-treated WT mice, but plays no apparent role in SNPinduced basal allodynia in saline-treated animals.

Because females have increased risk of developing migraine and $\mathrm{MOH}$, we tested whether infusion of sumatriptan for 6 days 

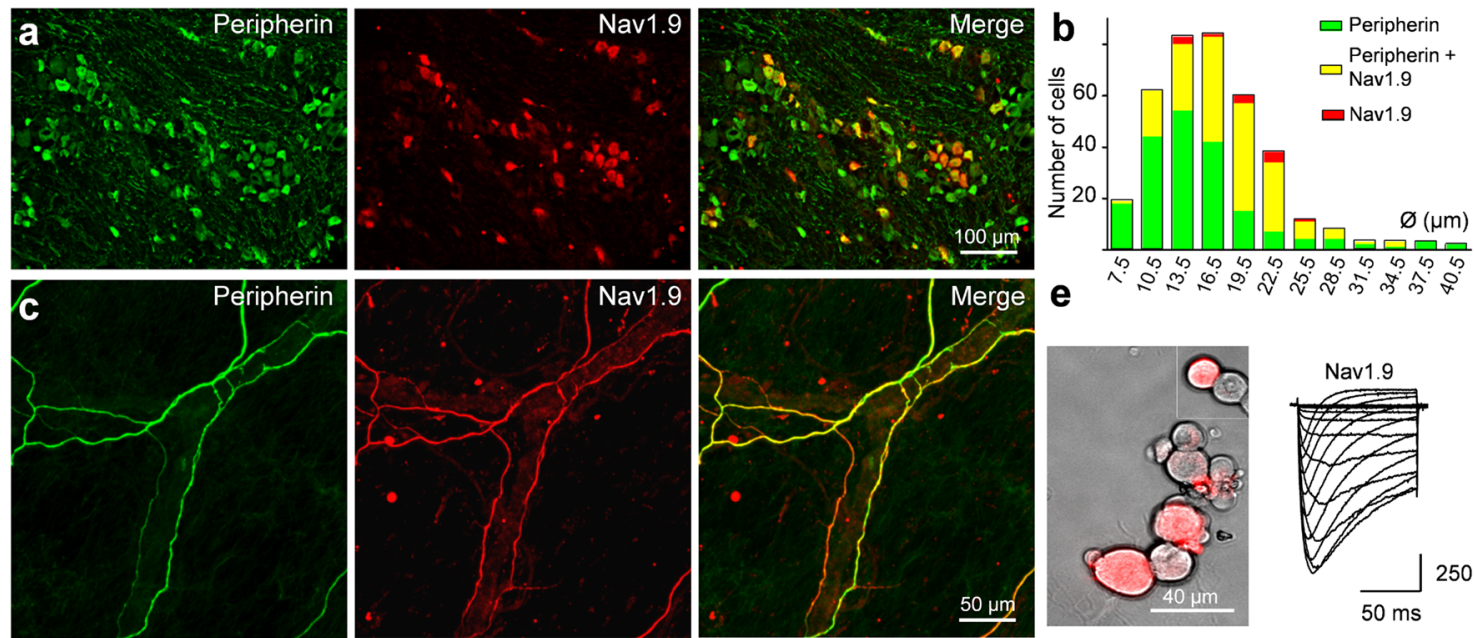

Nav1.9
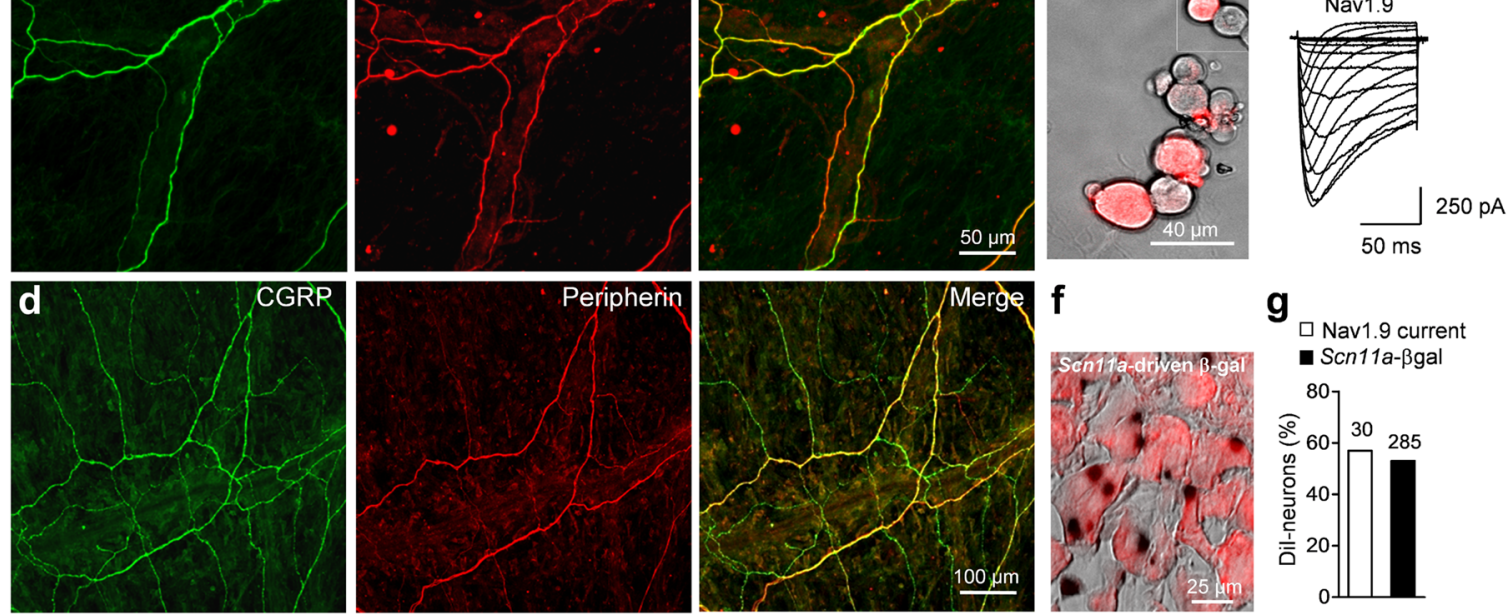

Fig. 1 Nav1.9 is expressed in meningeal nerve fibers and dural afferent neurons. a Cryosections of a mouse TG were double-labeled for peripherin and Nav1.9. Images are projections of seven consecutive optical sections spanning $9 \mu \mathrm{m}$. Rightmost panel: merged image. $\mathbf{b}$ Histogram showing the distribution of TG neurons immunopositive for peripherin (green), Nav1.9 (red), or both (yellow) as a function of cell body diameter ( $\varnothing$ ). Bin size $=3 \mu$ m. c Mouse whole-mount of dura mater was double-labeled for peripherin and Nav1.9. Images are projections of 16 consecutive optical sections spanning $22 \mu$ m. Rightmost panel: merged image. d Immunostaining for CGRP and peripherin in mouse dura mater. Images are projections of 21 consecutive optical sections spanning $23 \mu \mathrm{m}$. Rightmost panel: merged image. e Left panel: TG neurons cultured 2 days after Dil application through a cranial window in the parietal bone. This is a composite image of two different fields of the same culture dish indicated by a white box. Right panel: recording of Nav1.9 current in the retrogradely labeled TG neuron indicated by an arrow. The current was evoked by $100 \mathrm{~ms}$-voltage steps from -80 to $-5 \mathrm{mV}$ from a $\mathrm{Vh}$ of $-100 \mathrm{mV}$. CsFcontaining patch pipette solution. f Image from a Scn11alacZ mouse showing $\beta$-galactosidase expression (dark dots) in Dil + afferent neurons (red) from the TG. $\mathbf{g}$ Histogram showing the percentage of Dil+ afferent neurons exhibiting Nav1.9 current (patch clamp) or $\beta$-galactosidase (staining)

$(0.6 \mathrm{mg} / \mathrm{kg} /$ day $)$ produced mechanical hypersensitivity and latent sensitization to NO in WT female mice as observed for the opposite gender. Chronic sumatriptan produced a strong reduction in mechanical withdrawal thresholds of the hind paw relative to saline-treated WT female mice and to sumatriptantreated Nav1.9-/- female mice (Supplementary Fig. 2B). Injection of SNP $(0.03 \mathrm{mg} / \mathrm{kg})$ at day 21 once sensory thresholds had returned to pre-sumatriptan baseline values caused significantly stronger mechanical allodynia in sumatriptan-treated WT female mice compared to saline-treated WT female mice (Supplementary Fig. 2C). SNP-induced heightened mechanical allodynia was absent in sumatriptan-treated Nav1.9-/- female mice (Supplementary Fig. 2D), reaching similar amplitude to that caused by SNP in saline-treated Nav1.9-/- female mice (Supplementary Fig. 2D). This series of experiments shows that Nav1.9, as observed in male mice, had no role in SNP-induced allodynia in saline-treated animals, but contributes to the heightened SNP allodynia in sumatriptan-treated female mice.

SNP injection at day 21 was also found to reduce mean periorbital von Frey thresholds (periorbital allodynia) in sumatriptan-treated WT male mice compared with salinetreated WT male mice (Fig. 2e). Deletion of Nav1.9 significantly attenuated the SNP-induced periorbital allodynia in sumatriptantreated animals (Fig. 2e). Altogether, these data indicate that Nav1.9 contributes to NO-induced cephalic and extracephalic tactile allodynia in $\mathrm{MOH}$ mice.
Lack of NO-induced visual and auditory symptoms in Nav1.9 KO mice. Besides pain, disabling symptoms of $\mathrm{MOH}$ often include photophobia and/or phonophobia ${ }^{28}$. To evaluate lightaversive behavior (photophobia) of mice after SNP injection, we used the light-dark transition test ${ }^{29}$. Two hours after SNP injection, sumatriptan-treated WT mice spent significantly more time in the dark chamber relative to saline-treated WT mice (Fig. 3a). Sumatriptan-treated Nav1.9-/- mice challenged with SNP showed no signs of photophobia as the animals spent no more time in the dark box than saline-treated WT or Nav1.9-/mice treated likewise (Fig. 3a).

We further quantified the sound sensitivity (phonophobia) of mice following SNP injection by measuring the intensity of sound required to induce the acoustic startle reflex (ASR). We exposed mice to pseudo-random order of 100 ms-long white noise bursts ranging from 60 to $120 \mathrm{~dB}$ SPL at $10 \mathrm{kHz}$. The ASR threshold of saline-treated WT mice injected with SNP was $97 \pm 2 \mathrm{~dB}$ SPL $(n=7)$, which was significantly different than that of sumatriptantreated WT mice, which showed a lower ASR threshold of $90 \pm 1.5$ dB SPL $(n=6)$ (Fig. 3b). Because one decibel equals 10 times the common logarithm of the power ratio, a decrease in $\sim 10 \mathrm{~dB}$ SPL in ASR corresponds to a 10 -fold increase in sound sensitivity. SNP induced no changes in ASR threshold in sumatriptan-treated Nav1.9 $9^{-/}$mice compared to saline-treated Nav1.9 ${ }^{-/-}$mice (Fig. 3b). We then examined the prepulse inhibition (PPI) of the ASR. This response provides an operational measure of 


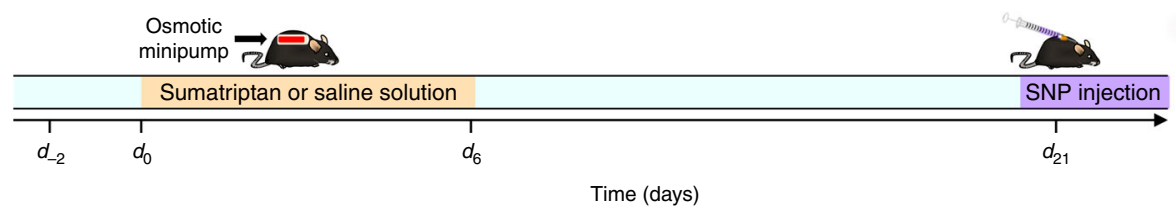

a

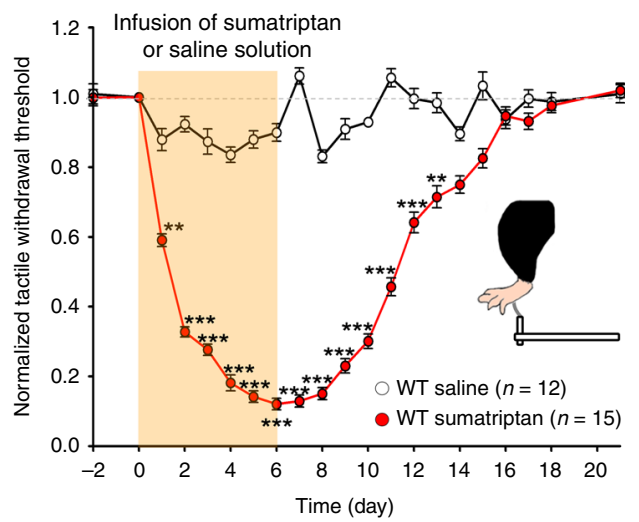

C

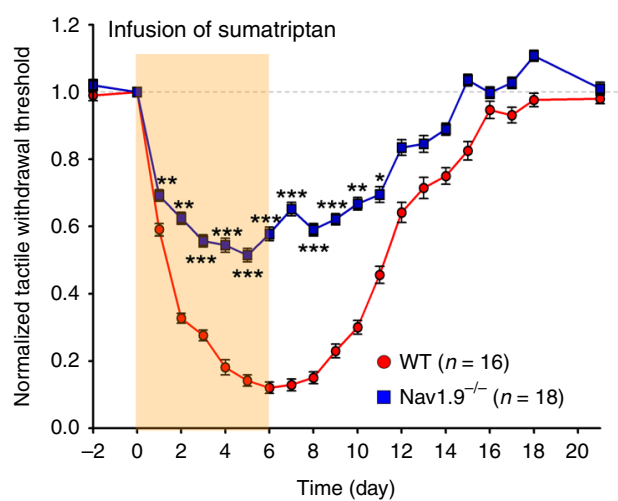

b SNP or

$$
\text { vehicle inj. }
$$

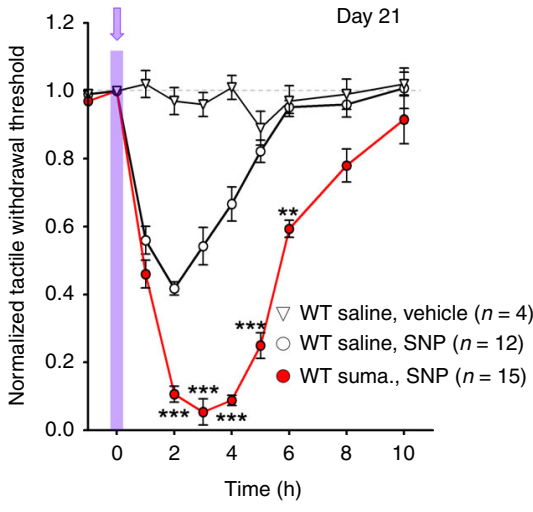

d SNP inj.

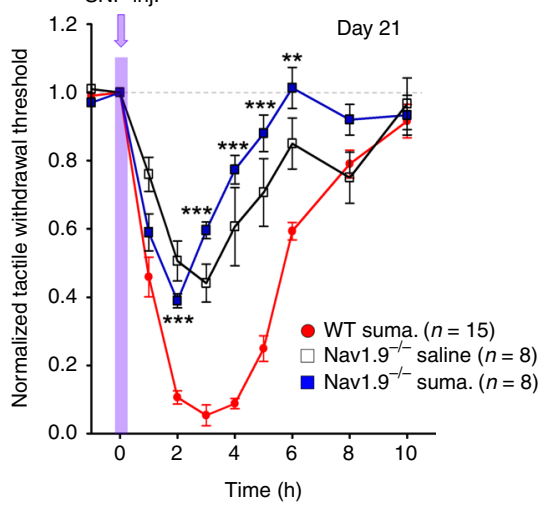

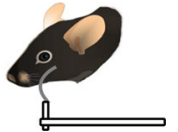

$\square$ WT saline $(n=8)$

WT suma. $(n=6)$

- Nav1. $9^{-1-}$ suma. $(n=6)$

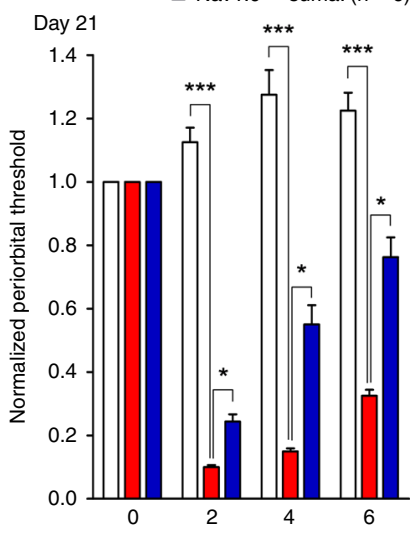

Post-SNP injection time (h)

Fig. 2 Deletion of Nav1.9 prevents NO-induced generalized allodynia in $\mathrm{MOH}$ mice. a Infusion of sumatriptan (0.6 mg/kg/day), but not saline solution $(0.9 \%)$, decreases withdrawal thresholds to tactile stimuli applied to the hind paws of WT mice. Hind paw withdrawal threshold was tested using von Frey filaments (inset). ${ }^{\star \star} p<0.01,{ }^{\star \star \star} p<0.001$ compared to saline with Mann-Whitney non-parametric test. Top inset: schematic of mouse treatment over time. b Changes in mechanical withdrawal thresholds of the hind paws induced by injection of SNP $(0.03 \mathrm{mg} / \mathrm{kg})$ in WT mice pre-treated (red symbols) or not (open circles) with sumatriptan. Data illustrated depict SNP responses 21 days after minipump implantation. ${ }^{\star \star} p<0.01,{ }^{\star \star \star} p<0.001$ compared to WT saline, SNP with Mann-Whitney non-parametric test. c Hind paw withdrawal responses of sumatriptan-treated Nav1.9-/- mice compared with sumatriptan-treated WT littermates. ${ }^{\star} p<0.05,{ }^{\star \star} p<0.01,{ }^{\star \star \star} p<0.001$ compared to WT with Mann-Whitney non-parametric test. $\mathbf{d}$ Comparison of SNPinduced changes in hind paw withdrawal thresholds in sumatriptan-treated WT mice (red symbols), saline-treated Nav1.9-/- mice (open squares) and sumatriptan-treated Nav1.9-/- mice (blue squares). All tests were made at day $21 .{ }^{\star \star} p<0.01,{ }^{\star \star \star} p<0.001$ compared to WT sumatriptan with two-way ANOVA followed by Student-Newman-Keuls test. e Normalized periorbital withdrawal threshold plotted as a function of time after SNP injection in salinetreated WT mice (open bars), sumatriptan-treated WT mice (red bars) and sumatriptan-treated Nav1.9-/- mice (blue bars). Data illustrated depict SNP responses 21 days after pump implantation. ${ }^{\star} p<0.05,{ }^{\star \star \star} p<0.001$; two-way ANOVA followed by Student-Newman-Keuls test

sensorimotor gating reflecting the sensitization of mice to weak sounds ${ }^{30}$. PPI was measured as the innate reduction of the startle reflex induced by a weak pre-stimulus sound (Fig. 3c). Following SNP injection at day 21 , the PPI value was $18.5 \pm 2 \%$ in salinetreated WT mice but reached $42.4 \pm 2.5 \%$ in sumatriptan-treated WT mice (Fig. 3c, d), indicating sensitization to sounds. By contrast, the PPI value was not significantly different in salinetreated versus sumatriptan-treated Nav1.9 $9^{-/}$mice (Fig. $3 \mathrm{c}, \mathrm{d}$ ).

Together, these data indicate that Nav1.9 is essential to the development of NO-induced symptoms of central sensitization observed in $\mathrm{MOH}$ mice.

Triptan overuse promotes coupling of NO-cGMP to Nav1.9 channels. We investigated the molecular basis that promotes increased sensitivity of $\mathrm{MOH}$ mice to NO. Given the difficulty to unambiguously isolate Nav1.9 from Nav1.8 currents when using CsCl-based pipette solution ${ }^{11,13,14}$, retrogradely labeled dural afferent neurons were studied from Nav1.8 $8^{-/}$mice. We provided evidence that inactivation of the Scn10a gene encoding Nav1.8 did not affect sumatriptan-induced latent sensitization and hypersensitivity to SNP in $\mathrm{MOH}$ mice (Supplementary Fig. 3).

Nav1.9 current recorded in dural afferent Nav1.8 $8^{-/-}$neurons cultured at day 21 (i.e. 21 days after pump implantation) was identifiable from its slow activation kinetics and incomplete inactivation, producing 'persistent' TTX-resistant $\mathrm{Na}^{+}$currents. Neither the mean Nav1.9 peak current density (Fig. 4b), nor the level of Nav1.9 mRNA expression (at day 21) (Supplementary 
a

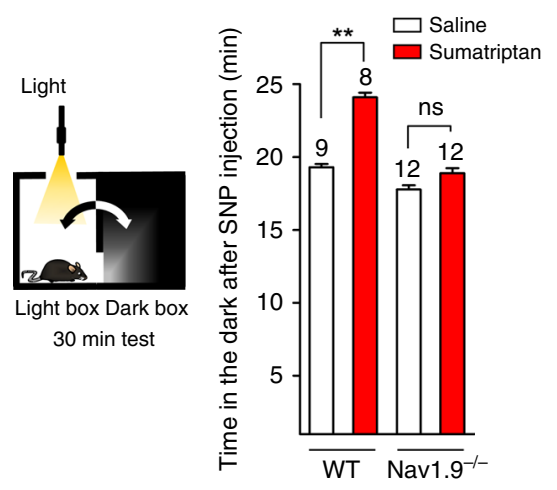

b

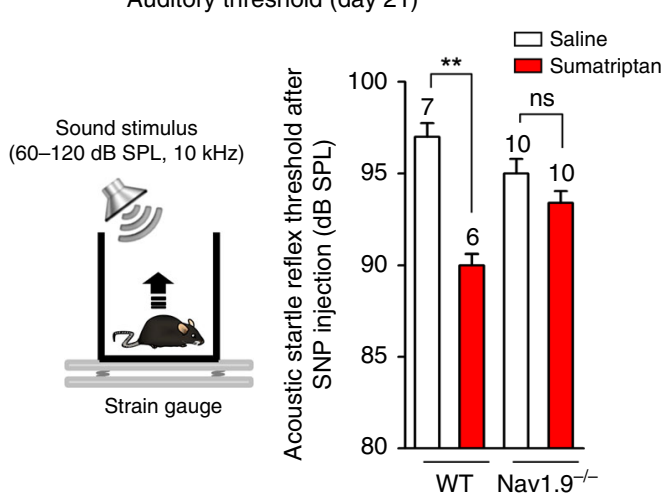

C

Sound sensitization after SNP injection (day 21)
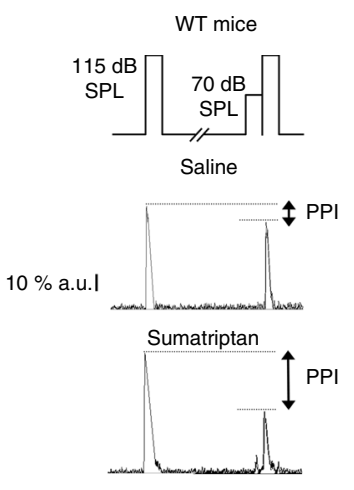
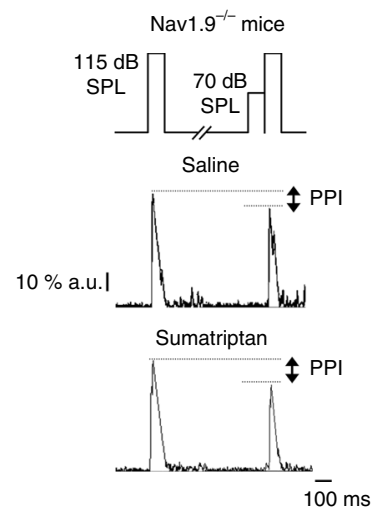

d

Sound sensitization after SNP injection (day 21)

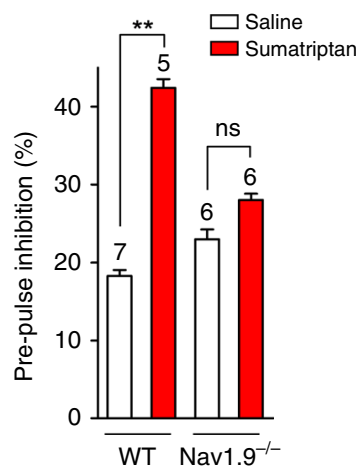

Fig. 3 Deletion of Nav1.9 prevents NO-induced photophobia and phonophobia in MOH mice. a Light sensitivity of sumatriptan-treated and saline-treated WT and Nav1.9-/- mice $2 \mathrm{~h}$ after injection of SNP $(0.03 \mathrm{mg} / \mathrm{kg})$ at day 21 . Visible light: $380-740 \mathrm{~nm}, 2600$ lx. Behavioral tests were made for a duration of 30 min. ns not significant; ${ }^{\star \star} p<0.01$; Mann-Whitney test. b ASR threshold in sumatriptan-treated and saline-treated WT and Nav1.9-/- mice $2 \mathrm{~h}$ after injection of SNP at day 21. ns not significant; ${ }^{\star \star} p<0.01$; Mann-Whitney test. $\mathbf{c}$ SNP-induced pre-pulse inhibition: the PPI was measured using the protocol illustrated (top panels) in saline- (middle panel) and sumatriptan- (bottom panel) WT and Nav1.9-/- mice $2 \mathrm{~h}$ after injection of SNP. a.u. arbitrary unit. d Comparison of the PPI in sumatriptan-treated or saline-treated WT and Nav1.9-/- mice $2 \mathrm{~h}$ after SNP injection. Tests were made at day 21. ns not significant; ${ }^{\star \star} p<0.01$; Mann-Whitney test

Fig. 4) was significantly different in TG neurons from salinetreated and sumatriptan-treated mice.

SNP $(1 \mathrm{mM})$ increased Nav1.9 peak current (measured at peak $I-V)$ by $232 \pm 11 \%(n=20)$ in dural afferent neurons from sumatriptan-treated Nav1.8 $8^{-1-}$ mice, whereas it had little effect $(72.7 \pm 4 \%, n=23)$ in control dural afferent neurons (Fig. $4 \mathrm{a}, \mathrm{b})$. Current-voltage relationships determined before and after SNP application in sumatriptan-treated dural neurons showed a $22 \mathrm{mV}$ negative shift in activation $V_{1 / 2}$ value (from $-11.3 \pm 1.8$ to $-32.7 \pm 1.02 \mathrm{mV}$ ). This shift was also associated with a $\sim 2$-fold increase in Nav1.9 maximum conductance (from -1.28 to -2.64 $\mathrm{nS} / \mathrm{pF}$ ) (Fig. 4c, d). By contrast, SNP induced a $\sim-6 \mathrm{mV}$ shift in dural afferent neurons from saline-treated mice (from $-14.47 \pm$ 1.3 to $-20.8 \pm 0.8 \mathrm{mV}$ ), which was not associated with change in $G_{\max }$ (Fig. 4d).

Because the cyclic guanosine monophosphate (cGMP) signal pathway plays an important role in NO signaling, we sought to determine the involvement of the soluble guanylyl cyclase (sGC) in the activation of Nav1.9. Application of methylene blue (100 $\mu \mathrm{M})$, a sGC inhibitor, abolished the effect of SNP on Nav1.9 in dural afferent neurons from sumatriptan-treated Nav1.8 ${ }^{-1-}$ mice (Supplementary Fig. 5). Consistently, the cell-permeable cGMP analog 8-Br-cGMP (1 mM) strongly increased Nav1.9 current and negatively shifted $V_{1 / 2}$ activation by $19 \mathrm{mV}$ (from -13.5 to $-32.5 \mathrm{mV}$ ) in saline-treated Nav1.8 ${ }^{-/-}$mice (Supplementary
Fig. 6A-C). These data indicate that NO-cGMP pathway activates Nav1.9 in sumatriptan-treated, but not in saline-treated dural afferent neurons.

Relief of PKA inhibition causes NO coupling to Nav1.9 in MOH mice. To probe the molecular changes in TG neurons at day 21 from sumatriptan-treated mice, we made qPCR analysis of cGMP-linked signaling molecules. Relative mRNA quantification showed there was a three-fold decrease of the transcript of protein kinase cAMP-activated catalytic subunit alpha (PKA-Ca) but no changes $(0.5<\mathrm{RQ}<2)$ for the cyclic nucleotide phosphodiesterases $3 a, 3 b$ and $5 a$ (PDE3a, PDE3b, and PDE5a), the sGC, the predominant receptor for NO, the Protein Kinase G type I (PKGI) and the Adenylyl Cyclase type III (AC-III) (Supplementary Fig. 7A). Pre-treatment of 8-Br-cAMP, a membrane permeable cAMP analog, inhibited cGMP-mediated activation of Nav1.9 in dural afferent neurons from saline-treated mice, although it had no effect per se on Nav1.9 (Supplementary Fig. 7B-D), indicating that cAMP can inhibit cGMP coupling to Nav1.9 in TG neurons. Consistently, we found that $\mathrm{DiI}^{+}$-dural afferent neurons showed strong staining for the catalytic PKA subunit phospho T197 antibody that recognizes the active form of the PKA protein (Supplementary Fig. 8A, B). In addition, the mean intensity (arbitrary unit) per pixel of PKA subunit phospho T197 staining was significantly $(p=0.03$, unpaired $t$-test) reduced from $131 \pm 4$ 
a

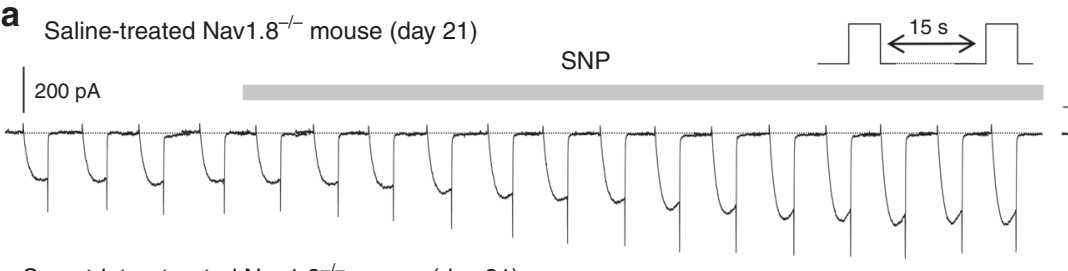

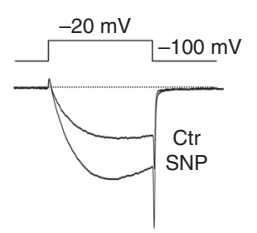

Sumatriptan-treated Nav1. $8^{-/-}$mouse (day 21)
SNP b

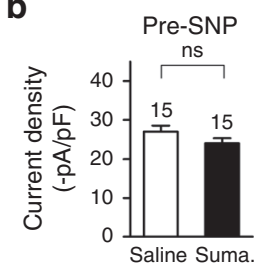

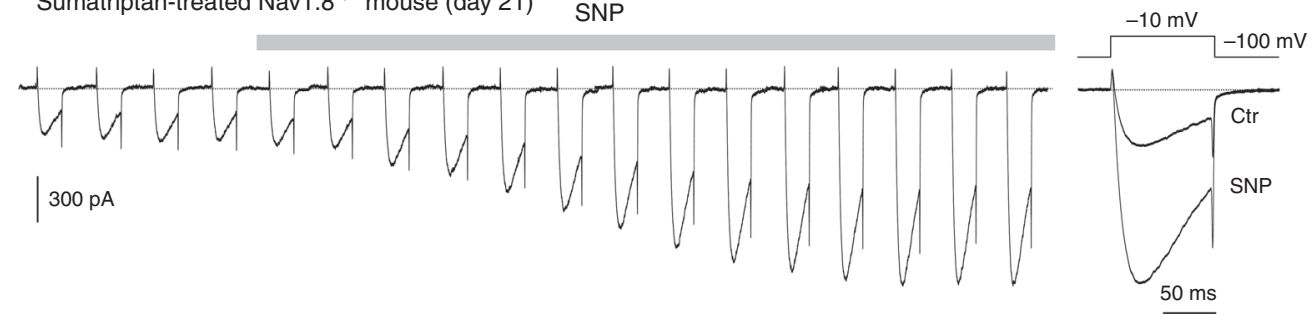

d

C

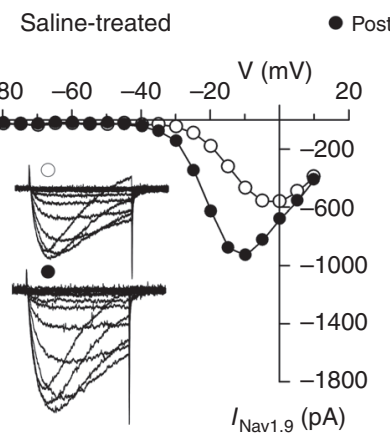

\section{Sumatriptan-treated}

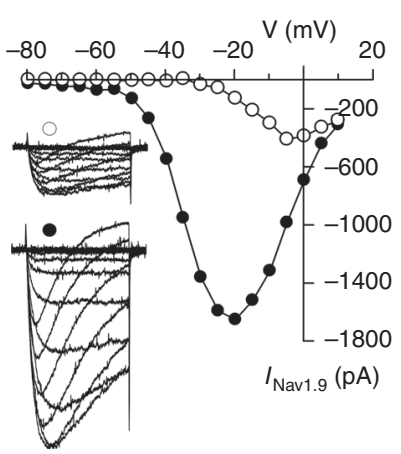

Saline-treated

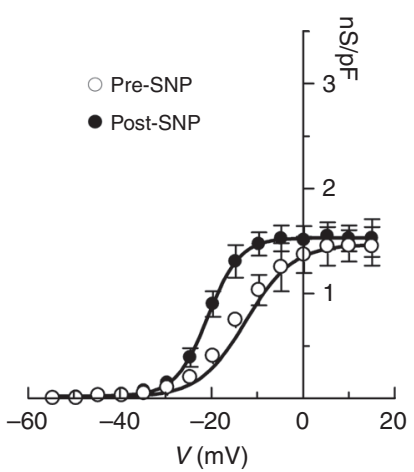

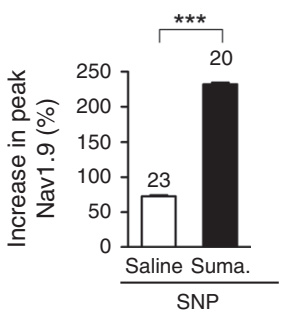

Sumatriptan-treated

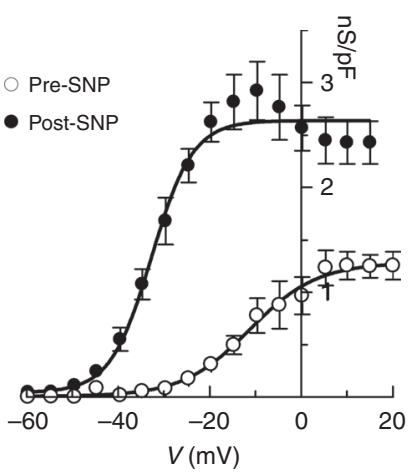

Fig. 4 Sumatriptan treatment promotes activation of Nav1.9 by NO. a Nav1.9 current exposed to $1 \mathrm{mM}$ SNP in dural afferent neurons from saline-treated (top panel) and sumatriptan-treated (bottom panel) Nav1.8 ${ }^{-/-}$mice. CsCl-only-based patch pipette solution. Right-most traces: superimposed Nav1.9 currents before and after SNP application. b Nav1.9 current density (top panel) in dural afferent neurons from saline-treated and sumatriptan-treated Nav1.8 $8^{-/-}$mice. ns, not significant; unpaired $t$-test. Bottom panel: mean increase in Nav1.9 peak current induced by SNP (1 mM) in dural afferent neurons from saline-treated and sumatriptan-treated Nav1.8 $8^{-/}$mice. ${ }^{\star \star *} p<0.001$; unpaired $t$-test. c Nav1.9 I-V determined in dural afferent neurons from salinetreated and sumatriptan-treated Nav1.8 ${ }^{-/-}$mice before and after SNP exposure. Insets: superimposed Nav1.9 current traces evoked by voltage steps from -80 to $+10 \mathrm{mV}$ from a $\mathrm{Vh}$ of $-100 \mathrm{mV}$. Note that not all traces are shown for clarity sake. $\mathbf{d}$ Activation curves for Nav1.9 current determined in Dil ${ }^{+}$-dural neurons before and after SNP application. Boltzmann fits gave $V_{1 / 2}$ values of $-14.47 \pm 1.3$ and $-20.8 \pm 0.8 \mathrm{mV}$ before and after SNP in saline-treated Nav1.8 $8^{-/}$mice $(n=11)$ and of $-11.3 \pm 1.8$ and $-32.7 \pm 1.02 \mathrm{mV}$ before and after SNP in sumaptriptan-treated Nav1.8 $8^{-/-}$mice $(n=9)$, respectively. All data collected from neurons cultured at day 21

( $n=44 \mathrm{DiI}^{+}$-neurons) in control mice injected with saline solution to $118 \pm 5\left(n=50 \mathrm{DiI}^{+}\right.$-neurons) in mice treated with sumatriptan (data not shown). Changes in PKA expression in TGs from sumatriptan-treated mice $(n=8)$ were also evaluated by quantifying band intensities on phospho T197 PKA blots and comparing to blot band intensities in saline-treated mice $(n=8)$ as controls. Densitometry analysis of background-subtracted blots from $20 \mu \mathrm{g}$ of total lysate showed a $22 \%$ decrease in phospho T197 PKA expression in sumatriptan-treated mice versus control mice (Supplementary Fig. 8C). This decrease however did not reach significant level due to sample variability (Supplementary Fig. 8D).

Collectively, these data suggest that nitrergic activation of Nav1.9 in sumatriptan-treated mice may result from a decrease in PKA activity in dural afferent neurons and subsequent relief of PKA-mediated inhibition of NO-Nav1.9 coupling.

Nav1.9-mediated hyperexcitability causes central sensitization. Firing activity of retrogradely labeled dural afferent neurons was studied at day 21. SNP-mediated Nav1.9 activation converted phasic discharges into multi-action potential (AP) responses in 78.5\% of dural afferent neurons from sumatriptan-treated WT mice (Fig. $5 \mathrm{a}-\mathrm{c}$ ). In addition, SNP reduced by $35 \%$ the current threshold for firing in neurons from sumatriptan-treated WT mice (Fig. 5d, e). By contrast, SNP had no significant effects on the firing response and AP current threshold in dural afferent neurons from saline-treated WT mice (Fig. 5c, d). Moreover, SNP caused no changes in excitation or AP current threshold in dural afferent neurons from sumatriptan-treated Nav1.9-/- mice (Supplementary Fig. 9).

We tested whether Nav1.9-mediated hyperexcitability regulates the secretion of CGRP, a key player in headache pathogenesis (Fig. 6a). SNP, at low concentrations, had no significant effects on basal secreted CGRP levels in TG cultures from saline-treated WT mice but enhanced CGRP secretion (+70\%) in TG cultures from sumatriptan-treated WT mice. Enhanced secretion of CGRP by SNP was not observed in TG cultures from sumatriptantreated Nav1.9-/- mice (Fig. 6a).

The in vivo consequence of Nav1.9-dependent CGRP secretion was tested on SNP-mediated extracephalic mechanical allodynia at day 21 using the CGRP antagonist $a-C_{\text {CGR }}$-37. Injection of 
a

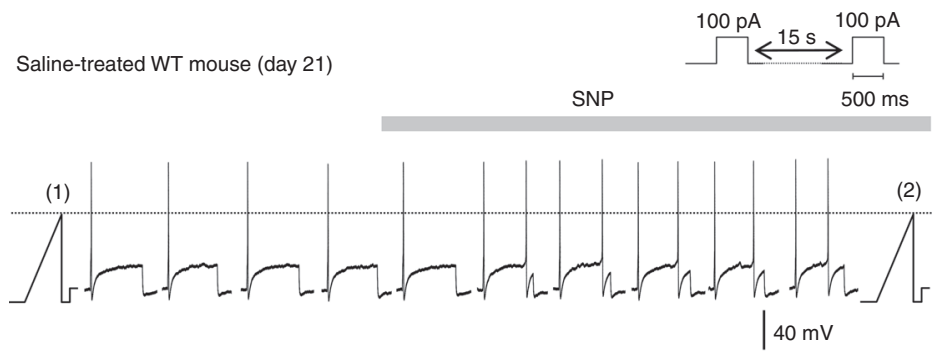

Sumatriptan-treated WT mouse (day 21)

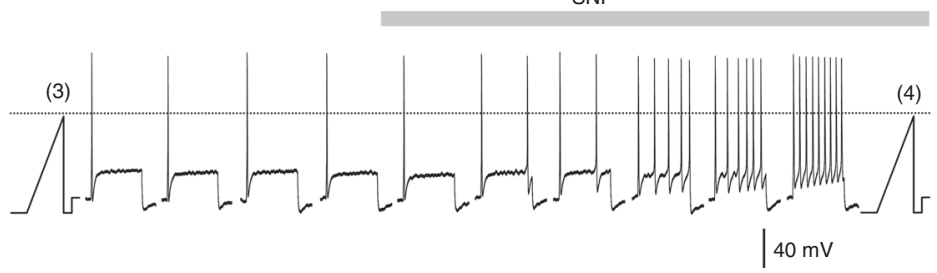

C

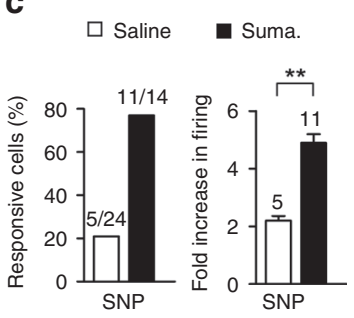

d

Saline-treated WT mouse
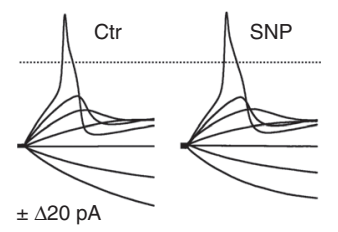

Sumatriptan-treated WT mouse

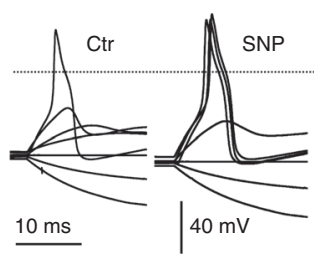

b
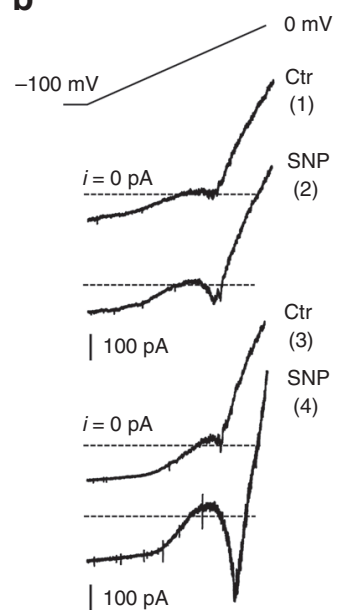

$100 \mathrm{pA}$

Fig. 5 Nav1.9 activation by NO lowers excitability threshold and enhances firing in dural afferent neurons. a Effect of SNP (1 mM) on Dil+ dural afferent neurons from saline-treated (upper panel) and sumatriptan-treated (bottom panel) WT mice. KCl-based intracellular solution throughout. b I-V relationships determined using a slow $(50 \mathrm{mV} / \mathrm{s})$ voltage ramp command in Dil ${ }^{+}$dural neurons illustrated in a before $(1,3)$ and during $(2,4) \mathrm{SNP}$ application. Note the activation of Nav1.9 (inwardly flowing current) by SNP (4). c Percentage of Dil+ neurons responding to SNP (left panel) and mean change in their firing rate (right panel). Protocol as in $\mathbf{a} .{ }^{\star \star} p<0.01$; Mann-Whitney test. $\mathbf{d}$ Generation of APs before and after SNP exposure in dural afferent neurons from saline-treated or sumatriptan-treated WT mice. Steady bias currents were used to maintain the neurons at - $65 \mathrm{mV}$. e Comparison of normalized current threshold for AP before and during SNP application in Dil+ dural neurons from saline-treated and sumatriptan-treated WT mice. ns not significant, ${ }^{*} p<0.05$ compared to saline-treated WT mice with Wilcoxon matched paired test. All data collected from TG neurons cultured at day 21

$\alpha_{-C_{1}}$ GRP $_{87}(1 \mathrm{mg} / \mathrm{kg})$, but not the vehicle, reduced SNP-induced extracephalic allodynia in sumatriptan-treated WT mice, indicating that released CGRP contributes to central sensitization (Fig. 6b). Importantly, injection of $a-\mathrm{CGRP}_{8-37}$ had no effects on residual SNP-induced allodynia in sumatriptan-treated Nav1.9-1animals (Fig. 6c), providing further evidence that Nav1.9 activation by SNP is a prerequisite for CGRP release from meningeal nociceptors.

Nav1.9 mediates vasodilatation and mast cell degranulation. Functional consequences of Nav1.9 activation on meningeal microcirculation was examined at day 21 using a laser Doppler blood perfusion scanner. SNP $(0.03 \mathrm{mg} / \mathrm{kg})$, injected through the jugular vein, caused a gradual increase of meningeal blood flow in sumatriptan-treated WT mice. No change was seen in saline-treated WT mice and in sumatriptan-treated Nav1.9-1mice (Fig. 6d, e). We further tested whether activation of meningeal nociceptors by SNP causes degranulation of dural mast cells (MCs) and pain amplification through a Nav1.9dependent mechanism. The MC stabilizing agent, sodium cromoglycate (SCG, $10 \mathrm{mg} / \mathrm{kg}$ i.p.), injected $30 \mathrm{~min}$ prior to SNP, significantly reduced both the intensity and duration of the SNP-induced heightened allodynia in sumatriptan-treated WT mice (Supplementary Fig. 10B) but had no significant effects on SNP-induced basal allodynia in saline-treated WT mice (Supplementary Fig. 10A) and on SNP-induced allodynia in sumatriptan-treated Nav1.9-/- mice (Supplementary Fig. 10C).

We finally sought to determine whether MC degranulation contributes to dural afferent terminal excitation through receptor-driven modulation of Nav1.9. Patch clamp recordings showed that the MC mediators histamine and PGE2 are powerful activators of Nav1.9 current in dural afferent neurons (Fig. 7). Superfusion of histamine and PGE2 increased peak Nav1.9 current by $100.5 \pm 25 \%$ and $233 \pm 40 \%$, respectively, in saline-treated Nav1.8 KO mice (Fig. 7a-g). Both mediators caused a substantial leftward shift in the activation curve of Nav1.9 (Fig. 7c, f) and strongly increased the firing rate of dural afferent neurons (Fig. 7h, i). These effects were seen irrespective of the mouse treatment (Fig. 7g). By contrast, CGRP, SP, and neurokinin A, which are potentially released by meningeal terminals, had no detectable effects on Nav1.9 current in dural afferent neurons from either saline-treated or sumatriptantreated mice (Supplementary Fig. 11A-D). Thus, some MC mediators have the capacity to activate Nav1.9, resulting in a feedforward loop that potentiates neurogenic inflammation and nociceptive transmission. Collectively, these results indicate that Nav1.9 acts as a hub in meningeal nociceptors and contributes to maladaptive nociceptive signal, neurogenic inflammation, meningeal vasodilatation, and mast cell degranulation (Fig. 8). 
a

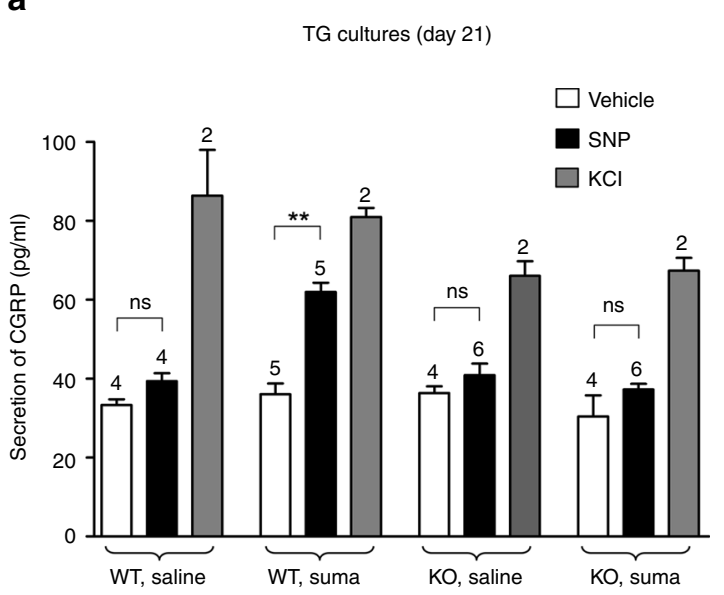

b

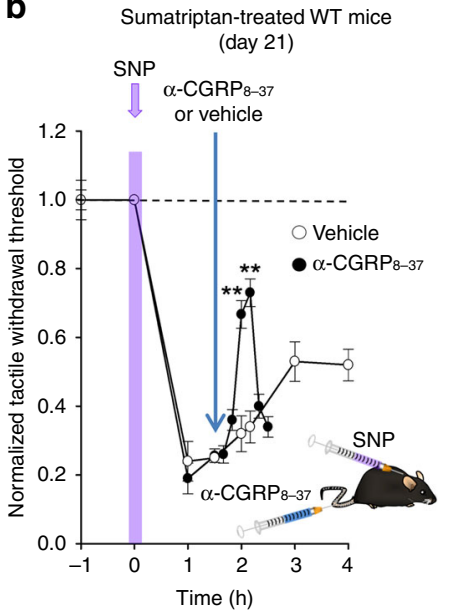

C Sumatriptan-treated Nav1. $9^{-/-}$mice

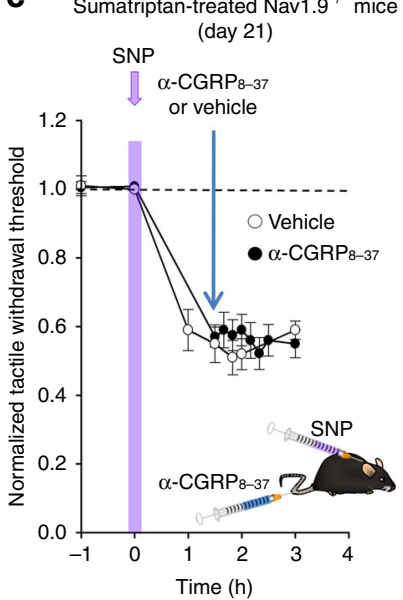

d

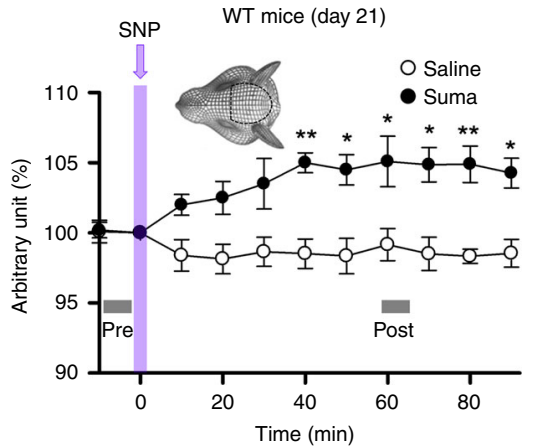

Saline-treated WT mouse

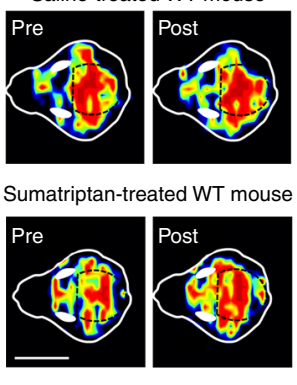

e

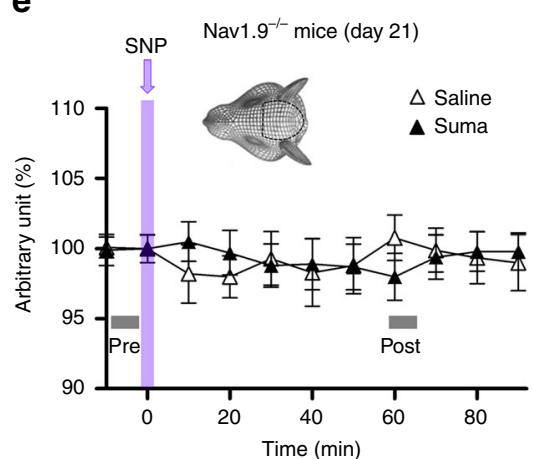

Saline-treated Nav1.9.-- mouse

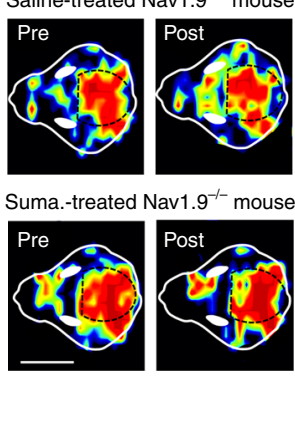

Fig. 6 Nav1.9 activation sustains CGRP release, which contributes to central sensitization and meningeal vasodilatation. a Effects of SNP (1 mM), HBSS (vehicle), and $\mathrm{KCl}(40 \mathrm{mM})$ on CGRP secretion in TG cultures from WT or Nav1.9-/- mice treated or not with sumatriptan. The $n$ number refers to the number of triplicates. ${ }^{\star \star} p<0.01$; Mann-Whitney test. b, c Effect of intravenous injection of $\alpha-\mathrm{CGRP}_{8-37}(1 \mathrm{mg} / \mathrm{kg})$ or its vehicle (NaCl $\left.0.9 \%\right)$ on SNP-

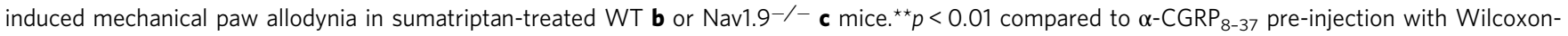
matched paired test ( $n=5$ per group). Behavioral tests were carried out at day 21. d-e SNP-induced meningeal blood flow changes in WT d and Nav1.9-/e mice, treated or not with sumatriptan. ${ }^{\star} p<0.05,{ }^{* \star} p<0.01$; Mann-Whitney test ( $n=6$ per group). Right panels: representative laser Doppler images taken before and $60 \mathrm{~min}$ after SNP injection. The blue color represents low perfusion areas, green and yellow refer to higher perfusion and red shows the highest microperfusion. Scale: $8 \mathrm{~mm}$

\section{Discussion}

How chronic exposure to abortive medication leads to $\mathrm{MOH}$ remains unclear. Our study suggests that chronic use of triptans induces $\mathrm{MOH}$ through abnormal activation of meningeal Nav1.9 by NO. Thus, triptan-overuse headache derives from Nav1.9 activation in the TG system, triggering pain facilitation through central and peripheral sensitization and inflammation in the meninges.

The complex pathophysiology behind $\mathrm{MOH}$ is still only partly known. Mechanisms involved may differ from one class of overused drug to another. Previous studies have shown that chronic use of opioids and triptans increases CGRP levels which is well known to be involved in neurogenic inflammation and headache pain ${ }^{8,31}$. Impaired diffuse noxious inhibitory controls ${ }^{6}$ and central sensitization are also seen in $\mathrm{MOH}$ patients ${ }^{4,32}$. Many of these phenomena are similar to mechanisms seen in dependence processes ${ }^{33,34}$.

Our approach to modeling $\mathrm{MOH}$ symptoms was the quantification of increased sensory sensitivity in response to $\mathrm{NO}$, one of the most common reported trigger for headache and migraine $8,9,35,36$. NO donors reliably trigger headache in normal subjects, but trigger migraine and severe pain in migraineurs ${ }^{36}$, and this condition is accompanied by an increase in blood levels of CGRP, which is directly linked to the severity of headache pain $^{37-39}$. In our study, $\mathrm{MOH}$ following $\mathrm{NO}$ infusion was evaluated using multiple headache-like responses in each individual animal, including cutaneous facial and extracephalic allodynia as well as aversion to light and noise. Our data show that chronic sumatriptan treatment of mice induces a state of dormant sensitization characterized by sharp, exacerbated sensory responses to $\mathrm{NO}^{8,9,40}$.

Clinical and preclinical studies have consistently demonstrated increased excitability of the TG system after medication overuse. TG neuronal hyperexcitability may facilitate the process of peripheral and central sensitization. We show that NO was capable of producing strong activation of dural afferent neurons in $\mathrm{MOH}$ mice and that sensory hypersensitivity and $\mathrm{MOH}$-associated symptoms were prevented by deleting Nav1.9 but not Nav1.8. Nav1.9 activation sustains the hyperexcitability of meningeal nociceptors and lowers the threshold response for afferent pain signaling. Importantly, coupling of NO to Nav1.9 was weak under normal conditions, consistent with the observation that deleting Nav1.9 had no impact on SNP-induced (basal) cutaneous allodynia in saline-treated mice. Thus, chronic sumatriptan treatment promotes coupling of NO to Nav1.9 channels in dural afferent neurons, thus lowering the threshold of the animal's susceptibility to respond to initiating factors of headache. Importantly, we found that hypersensitivity to SNP was greater in $\mathrm{MOH}$ female than in male mice, which parallels the sexual dimorphism reported in $\mathrm{MOH}$ and migraine in humans. 
a

Saline-treated Nav1. $8^{-/-}$mouse (day 21) b

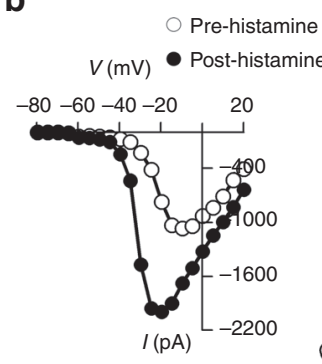

C

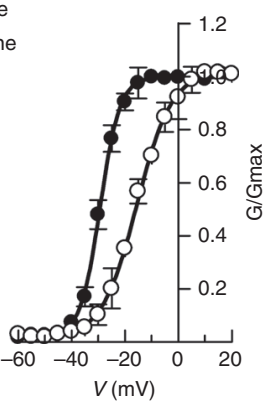

d

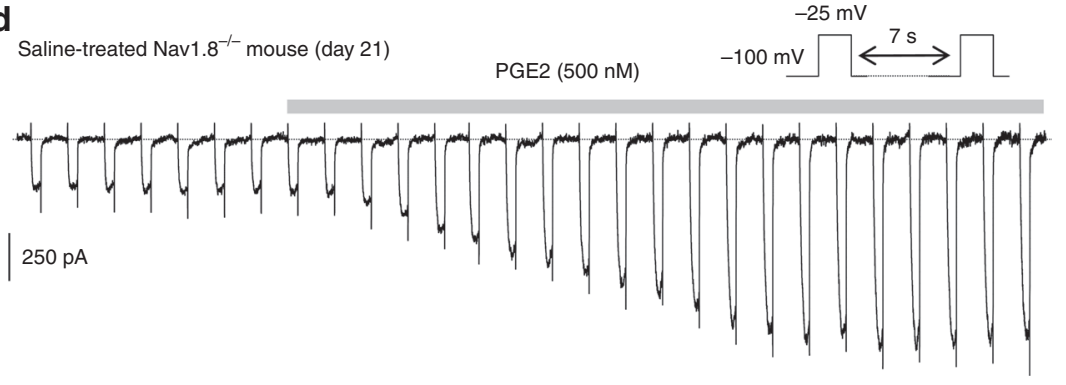

e

\section{f}

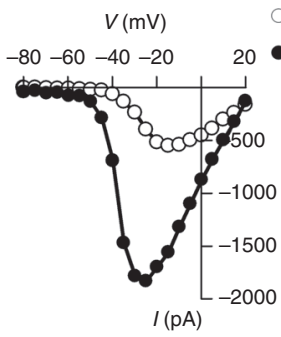

O Pre-PGE2

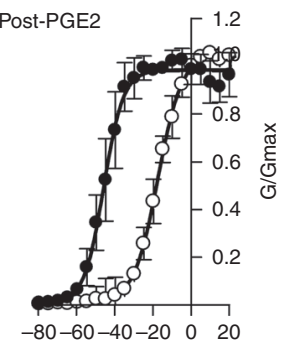

$V(\mathrm{mV})$

g

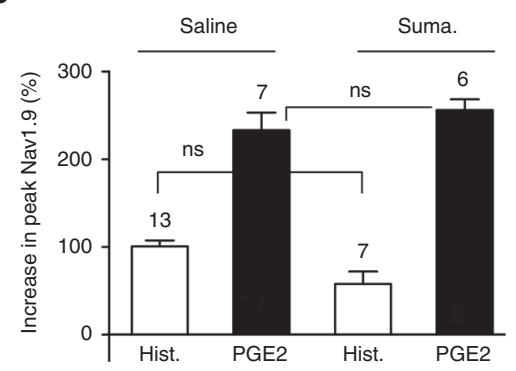

h

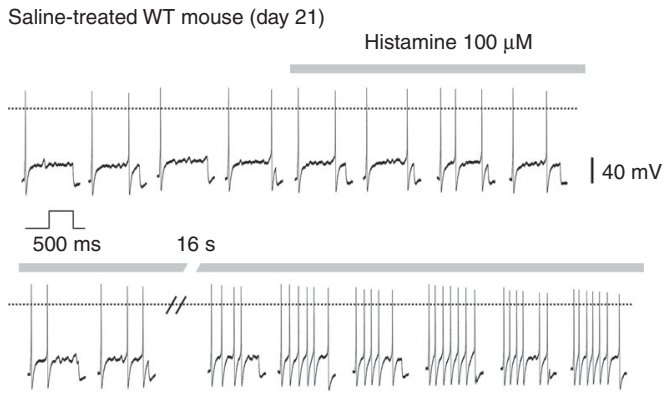

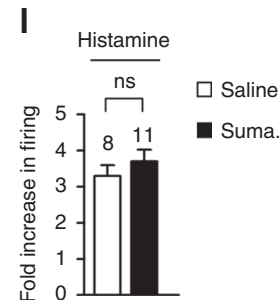

Fig. 7 Mast cell mediators activate Nav1.9 in dural afferent neurons. a Nav1.9 current challenged with $100 \mu$ M histamine in a Dil ${ }^{+}$dural afferent neuron from a saline-treated Nav1.8 $8^{-/}$mouse. CsCl-only-based patch pipette solution. $\mathbf{b}$ I- $V$ relationships from the cell depicted in $\mathbf{a}$ determined before and after histamine exposure. c Averaged activation curve for Nav1.9 determined before and after histamine application in Dil + neurons ( $n=13$ ). Single Boltzmann fits gave $V_{1 / 2}$ values of $-15 \pm 0.3$ and $-29.3 \pm 0.15 \mathrm{mV}$ before and after histamine application, respectively. $\mathbf{d}$ Nav1.9 current challenged with $500 \mathrm{nM}$ PGE2 in a $\mathrm{Dil}^{+}$dural afferent neuron from a saline-treated Nav1.8 $8^{-/-}$mouse. $\mathrm{CsCl}$-only-based patch pipette solution. e $1-V$ relationships from the cell depicted in d determined before and after PGE2 exposure. f Averaged activation curve for Nav1.9 determined before and after PGE2 application in Dil ${ }^{+}$dural afferent neurons $(n=7)$. Single Boltzmann fits gave $V_{1 / 2}$ values of $-17.3 \pm 0.6$ and $-45.6 \pm 1.1 \mathrm{mV}$ before and after PGE2 application, respectively. $\mathbf{g}$ Increase in Nav1.9 current (at peak $I / V$ as in $\mathbf{b}$ and $\mathbf{e}$ ) induced by histamine in Dil + neurons from saline-treated or sumatriptan-treated Nav1.8 ${ }^{-1-}$ mice. ns not significant, Mann-Whitney test. $\mathbf{h}$ Effects of histamine on a Dil ${ }^{+}$dural afferent neuron from a WT mouse treated with saline solution. Voltage responses were evoked by depolarizing pulses $(+50 \mathrm{pA})$ applied every $8 \mathrm{~s}$. $\mathrm{KCl}$-based intracellular solution. $\mathbf{i}$ Mean increase in firing of dural afferent neurons in response to histamine from WT mice treated with sumatriptan or the saline solution. ns not significant, Mann-Whitney test

What then favors Nav1.9 activation by NO/cGMP in sumatriptan-treated mice? PKA-Ca transcripts were found to be down-regulated in TGs from sumatriptan-treated animals at day 21 , suggesting that constitutive activity of PKA, in control animals, inhibits NO-Nav1.9 coupling. This is consistent with the observation that cAMP pretreatment inhibited cGMP-mediated activation of Nav1.9 in dural afferent neurons. How chronic stimulation of Gi-coupled 5HT1B/D receptors, which are notably expressed in TG neuron plasma membrane ${ }^{41}$, leads to long-term changes of PKA gene transcription remains to be determined.

Genetic deletion of Nav1.9 prevented SNP-induced meningeal vasodilatation, indicating that Nav1.9-dependent excitation of TG neurons was a prerequisite for this effect. Thus, Nav1.9 activation by SNP not only causes hyperexcitability of nociceptors but also triggers the release of vasoactive neuropeptides in the meninges. In vitro experiments further indicated that NO-induced release of
CGRP was prevented by deleting Nav1.9. These data indicate that Nav1.9-dependent release of CGRP, and possibly other neuropeptides including SP, plays a pivotal role in the vasodilatation of meningeal blood vessels. Our data however do not specify whether the vasodilatation contributes to $\mathrm{MOH}$-related symptoms or whether this is a side-phenomenon ${ }^{42}$. Therefore, if vasodilatation of meningeal arteries contributes to the propagation of the cascade of symptoms, it is likely in conjunction with other factors. CGRP is also poised to enhance headache pain by central mechanisms. Consistently, our results show that SNP-induced extracephalic allodynia was transiently alleviated by acute administration of the CGRP antagonist $\alpha-\mathrm{CGRP}_{8-37}$ in sumatriptan-treated animals. Because, extracephalic allodynia is a manifestation of central sensitization ${ }^{43}$, these data argue that Nav1.9-dependent CGRP release may also occurs at postsynaptic structures, such as the trigeminal nucleus caudalis, activity of which may sensitize thalamic neurons. These results call for an 
Sterile neurogenic inflammation

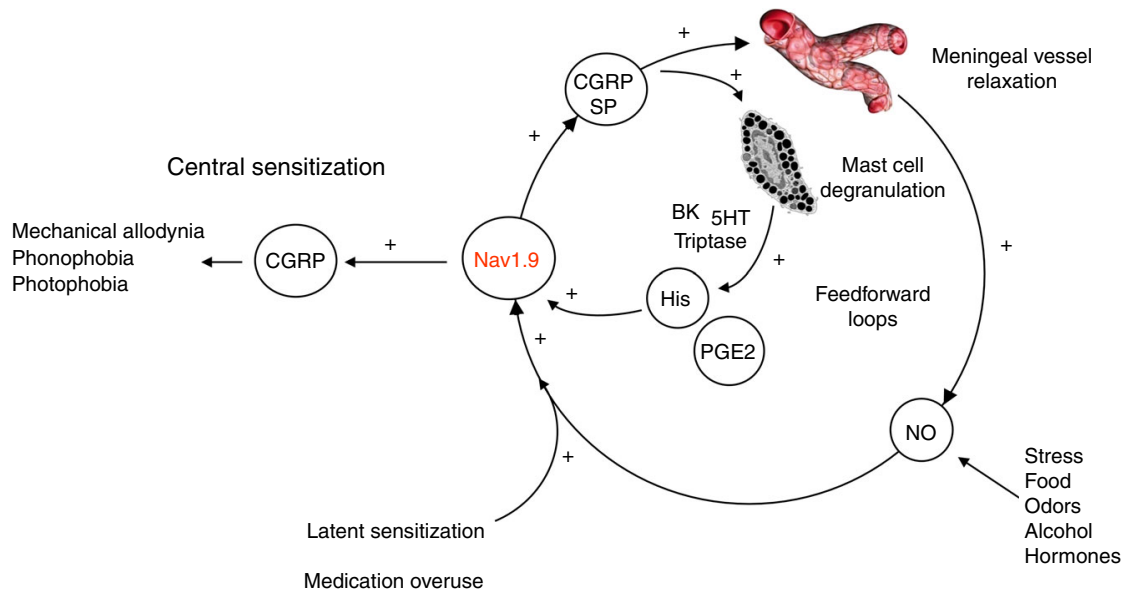

Fig. 8 Central role of Nav1.9 in $\mathrm{MOH}$ mechanisms. The following scenario summarizes the contribution of Nav1.9 to $\mathrm{MOH}$. NO, which may be released from different sources, activates Nav1.9 channels in dural afferent neurons from chronically treated mice with triptans. Nav1.9 activation by NO increases the excitability of meningeal nociceptors, which sensitizes central structures leading to extracephalic allodynia, photophobia, and phonophobia. Nav1.9dependent secretion of CGRP in the meninges, possibly in combination with other mediators, causes degranulation of resident mast cells. By releasing histamine and PGE2, MCs retro-excite meningeal nociceptors through Nav1.9 potentiation. Vasoactive peptides also contribute to vascular relaxation that may further facilitate endothelial (and possibly extravascular) NO production. The consequence is a vicious circle that leads to enhanced activation of meningeal nociceptors and maladaptive pain

early use of anti-Nav1.9 drugs that target meningeal nociceptors, before the development of central sensitization.

Our data demonstrate that Nav1.9-mediated release of neuropeptides from meningeal nociceptors could trigger degranulation of dural MCs. CGRP and SP, which are often co-released, are particularly important in this regard as they are known to stimulate MC degranulation in different systems ${ }^{44}$. Histamine, a major amine released from $\mathrm{MCs}^{45}$, and $\mathrm{PGE}_{2}$ another proinflammatory $\mathrm{MC}$ mediators ${ }^{46}$, are well-recognized migrainogenic substances known to induce pain upon infusion into human subjects diagnosed with migraine. Our data show that both substances cause a prominent increase of Nav1.9 and promote neuronal hyperexcitability, resulting in a vicious, selfreinforcing cycle of sterile inflammation and nociception (cf Fig. 8). Because MCs express a variety of 5-HT receptors, it is possible that chronic treatment with sumatriptan reinforces the possibility that MCs respond to peptide-induced degranulation. However, the reported effects of 5-HT on MCs have generally been found to be mediated by $5-\mathrm{HT} 1 \mathrm{~A}$ and not by $5 \mathrm{HT} 1 \mathrm{~B} / \mathrm{D}$ receptors 47 .

In conclusion, our study identifies NO-induced Nav1.9 channel activation as a triggering mechanism for $\mathrm{MOH}$-related symptoms. The way in which medication overuse transforms episodic migraine into chronic daily headache is still unknown. The picture that emerges from our study is that abnormal sensitivity of meningeal Nav1.9 channels to the migraine trigger NO may cause the headache phase in $\mathrm{MOH}$ patients. Activation of Nav1.9 may be a common denominator of overused drugs and migraine triggers. Therefore, the use of Nav1.9 channel inhibitors, in combination with sumatriptan or other headache medications, may represent a new acute and preventive option for migraine treatment.

\section{Methods}

Animals. This project was approved by the Institutional Review Board of the regional ethic committee. All animals were used in accordance with the European Community guiding in the care and use of animals (2010/63/UE). All efforts were made to minimize the number of animals used and their suffering. Mice (10-12week-old adult males and females, $\mathrm{C} 57 \mathrm{Bl} / 6 \mathrm{~J}$ background) used in this study were Nav1.9 $9^{-/-}$, Nav1.8 $8^{-/-}$(gifts from N.J. Wood, see refs. ${ }^{19,23}$ ) and their WT littermates. The Scn11a-GAL reporter mouse was previously described ${ }^{15}$. An IRES/ LacZpA cassette followed by a loxP/neo/loxP cassette was inserted into the end of exon 5 of the Scn11a gene.

Infusion of sumatriptan and injection of SNP. Alzet osmotic minipumps (model 1007D, Charles River, France) with a nominal flow rate of $0.5 \mu \mathrm{l} / \mathrm{h}$ for 6 days were used for drug infusion. The minipumps were implanted subcutaneously under anesthesia with isoflurane. The day of the pump implant was considered as day 0 . Drugs administered by infusion were sumatriptan $(0.6 \mathrm{mg} / \mathrm{kg} / \mathrm{day}$, Sigma, St. Louis USA) and its vehicle ( $\mathrm{NaCl}, 0.9 \%)$. SNP was injected subcutaneously into the loose skin over the neck at $0.03 \mathrm{mg} / \mathrm{kg}$. Infusion of sumatriptan/saline and injection of $\mathrm{SNP} /$ saline were made blind, e.g. the investigator was not aware of the content of the minipump, nor of the nature of the solution (SNP or vehicle) injected on day 21. Animals were randomly assigned to treatment groups.

Tactile sensory testing. Hind paw mechanical threshold was assessed using von Frey filaments (Bioseb, France) as described ${ }^{20}$. For facial testing, mice were subjected to a von Frey stimulus applied to the forehead surface, repeated three times (at minimum $30 \mathrm{~s}$ interval). The head withdrawal tactile sensory threshold was the lowest force to elicit withdrawal in 2 of 3 trials. Data points were normalized to the control sensory threshold values determined just before minipump implantation (i.e. at day 0) or before SNP injection (i.e. at day 21, H0).

Light aversive test. To evaluate the light-aversive behavior after SNP injection, we used a light-dark test. The light-aversion chamber consisted of two equally sized compartments $(10 \mathrm{~cm}$ width, $13 \mathrm{~cm}$ length, and $13 \mathrm{~cm}$ height), one painted white and lacking a top, the other painted black and fully enclosed. A corridor $(7.2 \mathrm{~cm}$ width, $7.2 \mathrm{~cm}$ length, and $13 \mathrm{~cm}$ height) connects the two compartments. Mice were acclimatized at least $1 \mathrm{~h}$ in their cages in the testing room and were allowed to explore the light-aversion chamber $10 \mathrm{~min}$ before testing. The light-aversive behavior was examined $2 \mathrm{~h}$ after SNP injection $(0.03 \mathrm{mg} / \mathrm{kg})$ and during $30 \mathrm{~min}$. The thermal-neutral fiber-optic source is located in the middle of white box and produces a light intensity of $2600 \mathrm{~lx}$ inside the lit area of the white chamber. Mouse transitions were interpreted to be a reflection of light aversion.

Acoustic startle threshold and prepulse inhibition tests. The Startle Reflex System (Bioseb, France) was used to measure the acoustic sensitivity of mice. The hardware consisted of an isolation cabinet that minimizes the effects of extraneous noise and vibrations. The startle chamber is situated in the center of the isolation cabinet $(250(W) \times 250(D) \times 250(H) \mathrm{mm})$ and a restrainer $(90 \times 30 \mathrm{~mm})$ was used to restrain minimally the animal during testing. Mice were acclimatized in the restrainer for $5 \mathrm{~min}$ per day, one week before the testing session. Mice were also acclimatized in their cages in the testing room, $2 \mathrm{~h}$ before the testing session. Acoustic stimuli for behavioral tests were based on previously published studies defining criteria applicable to mice at around 2 months of age. The waveforms 
generated by the mouse's movement during the startle response were analyzed using the Packwin software (Panlab, Inc., Harvard apparatus).

Two hours after SNP injection, the mouse was acclimatized for $5 \mathrm{~min}$ in the presence of a background noise level of $60 \mathrm{~dB}$ SPL. The mouse was then exposed to random trials of white noise bursts ranging from 60 to $120 \mathrm{~dB}$ SPL at $10 \mathrm{kHz}$, frequency that falls within the most sensitive region of the mouse audiogram. Each sound intensity was presented three times. The ASR threshold was taken as the minimum intensity required to elicit a response in two out of third trials. The PPI is a reliable, robust quantitative phenotype that is useful for probing sensory functions. Testing consisted of a series of $115 \mathrm{~dB}$ SPL-test pulses immediately preceded or not by a prepulse of $70 \mathrm{~dB}$ SPL at $10 \mathrm{kHz}$. PPI was calculated as follows: PPI $(\%)=(1-($ startle response with prepulse $) /($ startle response without prepulse) $) \times 100$

Cultures of TG neurons. Mice were anesthetized at day 21 with isoflurane and killed by decapitation. TGs were dissected out and freed from their connective tissue sheaths. TG neurons were incubated in enzyme solution containing $2 \mathrm{mg} / \mathrm{ml}$ of collagenase IA (Sigma) for $45 \mathrm{~min}$ at $37^{\circ} \mathrm{C}$. The tissue was washed several times and triturated in Hanks' balanced salt solution. The resulting suspension was filtered $(70 \mu \mathrm{m}$ filters $)$ and centrifuged $(800 \times g$ for $5 \mathrm{~min})$ and plated on poly-L-lysine/ laminin $(0.05$ and $0.01 \mathrm{mg} / \mathrm{ml}$, respectively) coated Nunclon dishes. Culture medium was Dulbecco's modified Eagle's medium supplemented with $10 \%$ heat inactivated FCS, $100 \mathrm{U} / \mathrm{ml}$ penicillin-streptomycin, $2 \mathrm{mM}$ L-glutamine, $25 \mathrm{ng} / \mathrm{ml}$ nerve growth factor (NGF), and $2 \mathrm{ng} / \mathrm{ml}$ glial-derived neurotrophic factor (GDNF).

Patch clamp recordings. Patch pipettes had resistances of $2 \mathrm{M} \Omega$. Voltage clamp recordings of $\mathrm{Na}^{+}$currents used the following intracellular solutions: (CsF-containing, in $\mathrm{mM}$ ), $30 \mathrm{CsF}, 100 \mathrm{CsCl}, 10 \mathrm{HEPES}, 10 \mathrm{EGTA}, 8 \mathrm{NaCl}, 1 \mathrm{MgCl}_{2}, 1 \mathrm{CaCl}_{2}$, 4 MgATP, $0.4 \mathrm{Na}_{2} \mathrm{GTP}$ (pH 7.35, $300 \mathrm{mOsm} / \mathrm{l}$ ); (CsCl-based, in $\mathrm{mM}$ ), $130 \mathrm{CsCl}$, 10 HEPES, 10 EGTA, $8 \mathrm{NaCl}, 1 \mathrm{MgCl}_{2}, 1 \mathrm{CaCl}_{2}, 4 \mathrm{MgATP}, 0.4 \mathrm{Na}_{2} \mathrm{GTP}$ (pH 7.35, $300 \mathrm{mOsm} / \mathrm{l}$ ). The extracellular solution contained (in $\mathrm{mM}$ ): $60 \mathrm{NaCl}, 110$ sucrose, $3 \mathrm{KCl}, 1 \mathrm{MgCl}_{2}, 10$ HEPES, $2.5 \mathrm{CaCl}_{2}, 10$ glucose, 10 TEA-Cl, 0.0005 TTX, 1 amiloride, $0.05 \mathrm{La}^{3+}(\mathrm{pH} 7.4,305 \mathrm{mOsm} / \mathrm{l})$. For current clamp recording, the intracellular solution (KCl-based) consisted of $(\mathrm{mM})$ : $115 \mathrm{KCl}, 10 \mathrm{HEPES}$

10 EGTA, $8 \mathrm{NaCl}, 1 \mathrm{MgCl}_{2}, 1 \mathrm{CaCl}_{2}, 4 \mathrm{MgATP}, 0.4 \mathrm{Na}_{2} \mathrm{GTP}$. The extracellular solution consisted of (in mM) $131 \mathrm{NaCl}, 3 \mathrm{KCl}, 1 \mathrm{MgCl}_{2}, 10 \mathrm{HEPES}, 2.5 \mathrm{CaCl}_{2}$, 10 glucose ( $\mathrm{pH} 7.4,305 \mathrm{mOsm} / \mathrm{l})$. All chemicals were from Sigma-Aldrich, except TTX (Alomone Labs)

PCLAMP 9.2 (Axon Instruments Inc.) and PRISM 4.0 (GraphPad) software suites were used to perform linear and nonlinear fitting of data. Conductance-voltage curves were calculated from the peak current according to the equation $G=I /\left(V-E_{\text {rev }}\right)$, where $V$ is the test pulse potential and $E_{\text {rev }}$ the reversal potential calculated according to the Nernst equation. The activation curve $(G-V)$ was fitted using the Boltzmann function: $G / G_{\max }=1 /\left(1+\exp \left[\left(V_{1 / 2}-V\right) / k\right]\right)$, where $G / G_{\max }$ is the normalized conductance, $V_{1 / 2}$ is the potential of half-maximum channel activation, and $k$ is the steepness factor.

Tracer application onto the dura. Mice were anesthetized with isoflurane. Throughout surgery, the core temperature of the mouse was monitored and maintained by an homeothermic blanket system for rodents. Two small cranial windows were made in parietal bones and the retrograde nerve tracer DiI (DiI tissue labeling paste, Invitrogen) was applied onto the dura. The bone flaps were then replaced after the procedure with bone wax in order to prevent tracer spreading. Animals were euthanized for TG extraction 2 days after DiI application.

\section{Tissue preparation, immunostaining, and confocal imaging. For Nav1.9}

immunostaining, the tissues were cryoprotected in PBS containing $4 \%$ sucrose during $30 \mathrm{~min}$ and then incubated for at least $1 \mathrm{~h}$ in PBS plus $20 \%$ sucrose at $4{ }^{\circ} \mathrm{C}$. The TGs were frozen in OCT embedding matrix bathed in chilled isopentane. The TGs were then sagitally cryosectioned at $14-18 \mu \mathrm{m}$, transferred to SuperFrostPlus slides and stored at $-80^{\circ} \mathrm{C}$ until processed. Whole mount dura maters were transferred to SuperFrostPlus slides, frozen on dry ice and stored at $-80^{\circ} \mathrm{C}$ until processed. Primary antibodies used and dilutions were as follows: anti-peripherin 1/400 (mouse monoclonal, Millipore, Temecula, CA); anti-NF200 1/400 (chicken polyclonal, Aves Labs, Tigard, OR); anti-CD31 (1/400, rat polyclonal, BD Biosciences, Belgium); anti-Nav1.9 L23, (1/100, rabbit polyclonal) ${ }^{10}$; and anti-CGRP (1/ 300, goat polyclonal, AbCam).

For CD31 immunostaining, the tissues were fixed for $30 \mathrm{~min}$ at room temperature (RT) in Antigenfix (Microm Microtech, France) before being cryoprotected and frozen as described above. Slides with fresh frozen tissues or fixed with Antigenfix tissues were thawed at RT and then incubated for $90 \mathrm{~min}$ at RT in blocking solution containing 3\% BSA and $0.1 \%$ Triton X-100. Primary antibodies were diluted in PBS containing 3\% BSA and applied to tissues to be incubated overnight at $4{ }^{\circ} \mathrm{C}$ in sealed humidified chambers.

For CGRP immunostaining, the tissues were fixed in $4 \%$ PFA in PBS for $3 \mathrm{~h}$ at RT then cryoprotected in PBS containing $25 \%$ sucrose overnight at $4{ }^{\circ} \mathrm{C}$. The tissues were transferred to SuperFrostPlus slides, frozen and stored at $-80^{\circ} \mathrm{C}$ until processed. Slides with PAF-fixed tissues were thawed at RT and then incubated for $1 \mathrm{~h} 30 \mathrm{~min}$ at RT in blocking solution containing $5 \%$ fish gelatin and $0.2 \%$ Triton X-
100. CGRP antibodies were diluted in PBS containing 5\% fish gelatin and applied to tissues to be incubated overnight at RT in sealed humidified chambers.

After incubation with the primary antibodies, fixed and fresh frozen tissues were identically proceeded. Tissues were washed three times for $5 \mathrm{~min}$ in PBS and incubated for $45 \mathrm{~min}$ at RT with secondary antibodies diluted in blocking buffer. Secondary antibodies were: Alexa Fluor 647-conjugated donkey anti-mouse (1/400, Life Technologies), TRITC-conjugated donkey anti-rabbit (1/400, Jackson ImmunoResearch, Suffolk, UK), TRITC-conjugated donkey anti-rat (1/100, Jackson ImmunoResearch), Alexa Fluor 488-conjugated donkey anti-goat (1/200, Life Technologies). After six 5 min-washes in PBS, sections were mounted in Mowiol (Sigma-Aldrich). Images were acquired using a LSM 780 laser-scanning confocal microscope (Zeiss), initially processed using ZEN software (Zeiss) and later exported into Adobe Photoshop (Adobe Systems, San Jose, CA) for final processing.

Staining for beta-galactosidase enzyme activity. Evaluation of $\beta$-gal enzymatic activity was performed on transgenic mice that express $\beta$-gal at the Scn11a loci. Once dissected out, the TGs were slightly fixed 15 min in PBS containing 2\% PFA, $0.2 \%$ glutaraldehyde, and $2 \mathrm{mM} \mathrm{MgCl}_{2}$. The tissues were washed with two changes of PBS containing $2 \mathrm{mM} \mathrm{MgCl}_{2}$ for 5 and $8 \mathrm{~min}$. The staining is achieved with an overnight incubation of TGs in PBS added with $4 \mathrm{mM}$ Ferrocyanide, $4 \mathrm{mM}$ Ferricyanide, $2 \mathrm{mM} \mathrm{MgCl} 2,1 \%$ Tween- 20 , and $0.2 \mathrm{mg} / \mathrm{ml} \mathrm{X-gal} \mathrm{at} 37^{\circ} \mathrm{C}$. The TGs were finally post-fixed for $15 \mathrm{~min}$ in PBS containing $2 \%$ PFA, $0.2 \%$ glutaraldehyde, and $2 \mathrm{mM} \mathrm{MgCl}_{2}$, and washed four times in PBS. The TGs were cryoprotected in PBS containing $4 \%$ sucrose for $30 \mathrm{~min}$ and then incubated overnight in PBS plus $20 \%$ sucrose at $4{ }^{\circ} \mathrm{C}$. The TGs were then frozen in OCT embedding matrix (Cellpath, Hemel Hempstead, UK) bathed in chilled isopentane. TGs were then sagitally cryosectioned at $14-18 \mu \mathrm{m}$, transferred to SuperFrostPlus slides (Fisher Scientific, Houston, TX), dried at RT and mounted in Mowiol (Sigma-Aldrich).

CGRP staining in TGs from Scn11a-GAL reporter mice. $\beta$-gal enzymatic activity in TGs from Scn11a-GAL reporter transgenic mice was revealed as above, except that glutaraldehyde was omitted from the fixative solution. Once achieved, TGs were post-fixed for $2 \mathrm{~h}$ in PBS containing $2 \% \mathrm{PFA}$ and $2 \mathrm{mM} \mathrm{MgCl}_{2}$, washed four times in PBS and then cryoprotected in PBS containing 25\% sucrose overnight. TGs were then frozen in OCT embedding matrix bathed in chilled isopentane and sagitally cryosectioned at 14-18 $\mu \mathrm{m}$, transferred to SuperFrostPlus slides, dried at $\mathrm{RT}$ and stored at $-80^{\circ} \mathrm{C}$. Slides with TGs were thawed for $15 \mathrm{~min}$ at RT and then incubated for $1 \mathrm{~h} 30 \mathrm{~min}$ at RT in blocking solution containing 3\% BSA and $0.1 \%$ Tx-100. CGRP antibody (rabbit polyclonal \#PC205L, Millipore) were diluted at $1 /$ 200 in blocking buffer and applied to tissues to be incubated overnight at $4{ }^{\circ} \mathrm{C}$ in sealed humidified chambers. Tissues were washed three times for $10 \mathrm{~min}$ in PBS and incubated $40 \mathrm{~min}$ at RT in PBS containing 3\% BSA added with secondary antibodies Alexa Fluor 488-conjugated donkey anti-rabbit (1/200, Life Technologies). After successive 5 min washes in PBS, sections were mounted in Mowiol (Sigma-Aldrich). Images were acquired using a conventional fluorescence microscope (Zeiss Axio-observer) with constant acquisition settings.

Quantitation of anti-PKA immunostaining. The TG ganglia were carefully dissected out from $\mathrm{NaCl}$-treated and sumatriptan-treated mice whose dura were DiIstained (see above). The TGs were fixed in $4 \%$ PFA in PBS for $2 \mathrm{~h} 30 \mathrm{~min}$ at $4{ }^{\circ} \mathrm{C}$ then cryoprotected in PBS containing $25 \%$ sucrose overnight at $4{ }^{\circ} \mathrm{C}$. The TGs were then frozen in OCT embedding matrix bathed in chilled isopentane and stored at $-80^{\circ} \mathrm{C}$ until processed. The day before PKA staining, the TGs were sagitally cryosectioned at $14 \mu \mathrm{m}$, transferred to SuperFrostPlus slides and stored at $-80^{\circ} \mathrm{C}$ Slides with PAF TGs were thawed $15 \mathrm{~min}$ at RT and then incubated for $30 \mathrm{~min}$ at $\mathrm{RT}$ in blocking solution containing $3 \%$ fish gelatin and $0.05 \%$ saponin. PKA antibodies (rabbit polyclonal \#ab75991, AbCam) were diluted at 1/200 in blocking buffer and applied to tissues to be incubated overnight at $4{ }^{\circ} \mathrm{C}$ in sealed humidified chambers. Tissues were washed three times for $10 \mathrm{~min}$ in PBS and incubated for $3 \mathrm{~h}$ at RT in PBS containing 3\% fish gelatin added with secondary antibodies Alexa Fluor 488-conjugated donkey anti-rabbit (1/200, Life Technologies). After four 5 min washes in PBS, sections were stained with DAPI, washed once in PBS and mounted in Mowiol (Sigma-Aldrich).

In order to compare the fluorescence intensities of PKA immunostaining between NaCl-treated and sumatriptan-treated DiI-positive TG neurons, images were acquired using a LSM 780 laser-scanning confocal microscope (Zeiss) using constant acquisition settings. ImageJ software was used to measure intensities of fluorescence on planar projections of confocal raw images spanning $6 \mu \mathrm{m}$. Elliptical regions of interest encompassing the soma of each DiI-positive neuron were defined in the DAPI image to exclude staining of the nucleus. The measurements were repeated in an adjacent area out of the tissue and the resulting background intensities were subtracted from the soma fluorescence signal. Results are expressed as mean grey value intensity per pixel meaning the sum of grey values of all pixels in the ROI divided by the number of pixels.

PKA western blot analysis. TGs were collected at 21 days after sumatriptan or saline infusion (T0), scrapped in TE buffer (66 mM Tris pH 6.8, 2\% SDS, $10 \%$ glycerol, $0.1 \mathrm{M} \mathrm{DTT}$, and antiprotease $2 \times$ ) and sonicated $3 \times 5 \mathrm{~s}$. The protein 


\begin{tabular}{|c|c|c|}
\hline Gene & Accession no. & $\begin{array}{l}\text { Targeted sequence } \\
\left(5^{\prime}-3^{\prime}\right)\end{array}$ \\
\hline Nav1.7 & XM_006499033 & $\begin{array}{l}\text { f-CCTTGGCCCCATTAAATCTCT } \\
\text { r-TGCTCCTATGAGTGCGTTGAC }\end{array}$ \\
\hline Nav1.8 & XM_006511991 & $\begin{array}{l}\text { f-TTGACACAACCTCGCTCTATTCC } \\
\text { r-ATTTCACCCTGGGTCTTCTCTCA }\end{array}$ \\
\hline Nav1.9 & AF118044 & $\begin{array}{l}\text { f-CCCTTGTGAGTCTCGCTGAC } \\
\text { r-GGAGTGGCCGATGATCTTAAT }\end{array}$ \\
\hline $5 \mathrm{HT} 1 \mathrm{~B}$ & NM_010482.1 & $\begin{array}{l}\text { f-TCGTTGCCACCCTTCTTCTG } \\
\text { r-CGTGGTCGGTGTTCACAAAG }\end{array}$ \\
\hline $5 \mathrm{HT} 1 \mathrm{D}$ & NM_008309.4 & $\begin{array}{l}\text { f-GGGTCAATTCATCAAGAACACA } \\
\text { r-GCTTGGAAGCTCTGAGGTGT }\end{array}$ \\
\hline PDE3a & NM_018779 & $\begin{array}{l}\text { f-AATGGGACCACAAGAGAGGG } \\
\text { r-TTCACTCTGGGCTTGTGGAT }\end{array}$ \\
\hline PDE3b & NM_011055 & $\begin{array}{l}\text { f-AAACGATCGCCTCTTGGTCT } \\
\text { r-CCCAGGGTTGCTTCTTCATC }\end{array}$ \\
\hline PDE5a & NM_153422 & $\begin{array}{l}\text { f-GGAAATGGTGGGACCTTCACT } \\
\text { r-AAGAACAATACCACAGAATGCCA }\end{array}$ \\
\hline GC & NM_021896 & $\begin{array}{l}\text { f-TGTTCACCTCTGCAGGTCAT } \\
\text { r-CCACACAATATGCATCCCCG }\end{array}$ \\
\hline PKG2 & NM_008926.4 & $\begin{array}{l}\text { f-CGGAAGAGTGGAGCTTGTTA } \\
\text { r-AGCCATCGGCATCCAGAATTA }\end{array}$ \\
\hline AC3 & NM_138305 & $\begin{array}{l}\text { f- ACATGATGCCCACGATGATA } \\
\text { r-CAGCAGGATGAGCTGGAAG }\end{array}$ \\
\hline PKAcA & BC054834.1 & $\begin{array}{l}\text { f-GCAAAGGCTACAACAAGGC } \\
\text { r-ATGGCAATCCAGTCAGTCG }\end{array}$ \\
\hline GADPH & NM_008084 & $\begin{array}{l}\text { f-GCAAATTCAACGGCACA } \\
\text { r-CACCAGTAGACTCCACGAC }\end{array}$ \\
\hline
\end{tabular}

concentration was analyzed by spectrophotometer (Nanodrop); concentrations were normalized to $800 \mu \mathrm{g} / \mathrm{ml}$ in TE and bromophenol blue $(30 \mathrm{mM})$. Electrophoresis was performed via SDS-PAGE using bis/tris polyacrylamide gels $4-12 \%$ (Thermo Scientific). After blotting, nitrocellulose membranes were stained with ponceau red. For immunostaining, membranes were washed in TBST, blocked in blocking solution (5\% milk in TBST) and incubated with anti-PKA (rabbit monoclonal, 1:5000, Abcam, ab75991) and anti-GAPDH (mouse monoclonal, 1:5000, Millipore, MAB374) antibodies overnight at $4{ }^{\circ} \mathrm{C}$. The next day, the membranes were washed three times in TBST, incubated with secondary polyclonal-HRP-conjugated goat anti-rabbit (1:2000, Biorad, 1706515) or antimouse (1:2000, polyclonal, Biorad, 1706516) antibody for $1 \mathrm{~h}$ at RT and washed again three times in TBST. Afterward, immunoreactivity was visualized using ECL Western blotting substrate (Roche) and densitometrically quantified using a Gbox Analyzer (Syngene). Reported mean grey values were determined with ImageJ

CGRP secretion. TGs were dissected out at day 21 and cultured for 1 DIV. Cells were plated in flat bottom plates at a concentration of 15,000 cells per well. The supernatant of cultured TG neurons was removed for CGRP detection after $30 \mathrm{~min}$ incubation with $\mathrm{KCl}(40 \mathrm{mM}), \mathrm{SNP}(1 \mathrm{mM})$, or the vehicle $(0.9 \% \mathrm{NaCl})$. CGRP detection was made using an enzyme-linked immunoabsorbent assay kit (Spibio, Berlin Pharma, France). Samples were run in triplicate.

Laser Doppler blood perfusion scanning. Meningeal microcirculation was determined in isoflurane-anesthesized mice with a laser Doppler blood perfusion scanner (Periscan PIM-III, Perimed) through the closed cranium after removing the covering skin and the periosteum. Light particles that hit moving blood cells undergo a change in wavelength/frequency, while light particles which encounter static structures return unchanged. The scattered light that returns to the tissue surface is registered by a photodetector. The perfusion can be calculated since the magnitude and frequency distribution of the Doppler shifted light are directly related to the number and velocity of blood cells, but unrelated to their direction of movement. Although the measured parameter is flux, this signal is then processed to extract information about the microcirculatory blood flow. After a control period $(10 \mathrm{~min})$, SNP $(0.03 \mathrm{mg} / \mathrm{kg})$ was injected into the jugular vein catheter and the observation period lasted for $90 \mathrm{~min}$.

Quantitative real-time RT-PCR. Total RNA was extracted from TG tissue using the RNeasy ${ }^{\oplus}$ Plus minikit (Qiagen) and assessed on a NanoDrop ND-2000 spectrophotometer (Thermoscientific) for concentration $\left(A_{260}\right)$ and purity by OD ratios $\left(A_{260} / A_{280}\right.$, ranging between 2.0 and 2.2$)$. cDNA was synthesized by using $1.2 \mu \mathrm{g}$ total RNA from each sample with oligo-dT primers and Superscript III ${ }^{\circledR}$ (Invitrogen) in $20 \mu \mathrm{l}$ reactions according to the manufacturer's instructions. To assess the specificity of these reactions, no reverse transcriptase and no template controls were also generated. cDNA was stored at $-20^{\circ} \mathrm{C}$ before PCR detection. RT products were diluted and amplified in $12 \mu$ reactions using Kapa Sybr ${ }^{\circledast}$ Fast qPCR kits (Kapa Biosystems). GAPDH was selected as reference gene for normalization of the qPCR results. qPCR reactions were run in duplicate on a Applied Biosystems 7500 Fast Real-time PCR thermocycler using MicroAmp ${ }^{\circledR}$ Fast 96-well reaction plates. Thermocycling parameters were 40 cycles of $95^{\circ} \mathrm{C}$ for $3 \mathrm{~s}$ for denaturation and $60^{\circ} \mathrm{C}$ for $30 \mathrm{~s}$ for annealing and extension. Parameters and reaction conditions were identical for all sets of primers. To compare transcript levels between saline and sumatriptan TG samples, the relative quantification (RQ) method was used. First, the difference $(\Delta \mathrm{Ct})$ between the cycle threshold $(\mathrm{Ct})$ values of the target gene and the GAPDH gene was calculated. Then, the difference $(\triangle \triangle \mathrm{Ct})$ between the normalized values with or without sumatriptan was calculated: $\Delta \Delta \mathrm{Ct}=$ $\Delta \mathrm{Ct}_{\text {sumatriptan }}-\Delta \mathrm{Ct}_{\text {saline. }}$. Finally, to determine the ratio of expression levels in sumatriptan samples versus saline samples, we used the RQ formula as follows $\mathrm{RQ}=2^{-\Delta \Delta \mathrm{Ct}}$. Applied Biosystems 7500 Software v 2.0.6 was used for analysis. Primers used were described in Table 1.

Statistical analysis. All values are shown as mean \pm standard error of the mean (SEM) and $n$ represents the number of animals or cells examined. Except for some behavioral experiments, no statistical methods were used to pre-determine sample sizes but our sample sizes are similar to those reported in previous publications. Assessment of normality for sample $>14$ was tested using the

Kolmogorov-Smirnov or the D'Agostino-Pearson Omnibus K2 normality test. Tests for differences between two normally distributed populations were performed using two-tailed $t$-test. Small sample size lacks power to test normality, therefore we typically used non-parametric Mann-Whitney test and Wilcoxon's test to test for differences between two populations in small samples $(n \leq 15)$. Two-way (repeated measures) ANOVA followed by Student-Newman-Keuls multiple comparison procedure was used for experiments with multiple groups and two dependent variables. Figure legends specify which test was used for specific experiments. Significant levels were set at $p \leq 0.05$. Analysis used a combination of Clampfit 9.2 (Molecular Devices), Origin 7.0 (OriginLab), and PRISM 7.0 (GraphPad) softwares.

Reporting summary. Further information on research design is available in the Nature Research Reporting Summary linked to this article.

\section{Data availability}

The authors declare that all the data supporting the findings of this study are included in the article (or in the Supplementary material) and available from the corresponding author (P.D.)

Received: 4 December 2018 Accepted: 26 August 2019 Published online: 18 September 2019

\section{References}

1. Bigal, M. E., Lipton, R. B. \& Stewart, W. F. The epidemiology and impact of migraine. Curr. Neurol. Neurosci. Rep. 4, 98-104 (2004).

2. Headache Classification Committee of the International Headache Society (IHS). The International Classification of Headache Disorders, 3rd edition (beta version). Cephalalgia 33, 629-808 (2013).

3. Noseda, R. \& Burstein, R. Migraine pathophysiology: anatomy of the trigeminovascular pathway and associated neurological symptoms, cortical spreading depression, sensitization, and modulation of pain. Pain 154(Suppl. 1), S44-S53 (2013).

4. Ayzenberg, I. et al. Central sensitization of the trigeminal and somatic nociceptive systems in medication overuse headache mainly involves cerebral supraspinal structures. Cephalalgia 26, 1106-1114 (2006).

5. de Tommaso, M. et al. Abnormal brain processing of cutaneous pain in patients with chronic migraine. Pain 101, 25-32 (2003).

6. Perrotta, A. et al. Sensitisation of spinal cord pain processing in medication overuse headache involves supraspinal pain control. Cephalalgia 30, 272-284 (2010).

7. Kristoffersen, E. S. \& Lundqvist, C. Medication-overuse headache: a review. J. Pain. Res 7, 367-378 (2014)

8. De Felice, M. et al. Triptan-induced enhancement of neuronal nitric oxide synthase in trigeminal ganglion dural afferents underlies increased responsiveness to potential migraine triggers. Brain 133, 2475-2488 (2010).

9. De Felice, M. et al. Triptan-induced latent sensitization: a possible basis for medication overuse headache. Ann. Neurol. 67, 325-337 (2010).

10. Padilla, F. et al. Expression and localization of the Nav1.9 sodium channel in enteric neurons and in trigeminal sensory endings: implication for intestinal reflex function and orofacial pain. Mol. Cell. Neurosci. 35, 138-152 (2007). 
11. Scroggs, R. S. The distribution of low-threshold TTX-resistant Na+ currents in rat trigeminal ganglion cells. Neuroscience 222, 205-214 (2012).

12. Luiz, A. P., Kopach, O., Santana-Varela, S. \& Wood, J. N. The role of Nav1.9 channel in the development of neuropathic orofacial pain associated with trigeminal neuralgia. Mol. Pain 11, 72 (2015).

13. Dib-Hajj, S., Black, J. A., Cummins, T. R. \& Waxman, S. G. NaN/Nav1.9: a sodium channel with unique properties. Trends Neurosci. 25, 253-259 (2002).

14. Coste, B., Osorio, N., Padilla, F., Crest, M. \& Delmas, P. Gating and modulation of presumptive Nav1.9 channels in enteric and spinal sensory neurons. Mol. Cell. Neurosci. 26, 123-134 (2004).

15. Maingret, F. et al. Inflammatory mediators increase Nav1.9 current and excitability in nociceptors through a coincident detection mechanism. J. Gen. Physiol. 131, 211-225 (2008)

16. Baker, M. D., Chandra, S. Y., Ding, Y., Waxman, S. G. \& Wood, J. N. GTPinduced tetrodotoxin-resistant $\mathrm{Na}^{+}$current regulates excitability in mouse and rat small diameter sensory neurons. J. Physiol. 548, 373-382 (2003).

17. Dib-Hajj, S. D., Black, J. A. \& Waxman, S. G. NaV1.9: a sodium channel linked to human pain. Nat. Rev. Neurosci. 16, 511-519 (2015).

18. Ritter, A. M., Martin, W. J. \& Thorneloe, K. S. The voltage-gated sodium channel Nav1.9 is required for inflammation-based urinary bladder dysfunction. Neurosci. Lett. 452, 28-32 (2009).

19. Amsalem, M., Poilbout, C., Ferracci, G., Delmas, P. \& Padilla, F. Membrane cholesterol depletion as a trigger of Nav1.9 channel-mediated inflammatory pain. EMBO J. 37, e97349 (2018)

20. Lolignier, S. et al. Nav1.9 channel contributes to mechanical and heat pain hypersensitivity induced by subacute and chronic inflammation. PLOS ONE 6 , e23083 (2011).

21. Amaya, F. et al. The voltage-gated sodium channel $\mathrm{Na}(\mathrm{v}) 1.9$ is an effector of peripheral inflammatory pain hypersensitivity. J. Neurosci. 26, 12852-12860 (2006).

22. Priest, B. T. et al. Contribution of the tetrodotoxin-resistant voltage-gated sodium channel NaV1.9 to sensory transmission and nociceptive behavior. Proc. Natl Acad. Sci. USA 102, 9382-9387 (2005).

23. Lolignier, S. et al. The Nav1.9 channel is a key determinant of cold pain sensation and cold allodynia. Cell Rep. 11, 1067-1078 (2015)

24. Huang, J. et al. Gain-of-function mutations in sodium channel $\mathrm{Na}(\mathrm{v}) 1.9$ in painful neuropathy. Brain 137, 1627-1642 (2014).

25. Zhang, X. Y. et al. Gain-of-function mutations in SCN11A cause familial episodic pain. Am. J. Hum. Genet. 93, 957-966 (2013).

26. Leipold, E. et al. A de novo gain-of-function mutation in SCN11A causes loss of pain perception. Nat. Genet. 45, 1399-1404 (2013).

27. Han, C. et al. Familial gain-of-function $\mathrm{Na}(\mathrm{v}) 1.9$ mutation in a painful channelopathy. J. Neurol. Neurosurg. Psychiatry 88, 233-240 (2017).

28. Edvinsson, L. \& Uddman, R. Neurobiology in primary headaches. Brain Res. Rev. 48, 438-456 (2005).

29. Markovics, A. et al. Pituitary adenylate cyclase-activating polypeptide plays a key role in nitroglycerol-induced trigeminovascular activation in mice. Neurobiol. Dis. 45, 633-644 (2012).

30. Geyer, M. A., McIlwain, K. L. \& Paylor, R. Mouse genetic models for prepulse inhibition: an early review. Mol. Psychiatry 7, 1039-1053 (2002).

31. Belanger, S., Ma, W., Chabot, J. G. \& Quirion, R. Expression of calcitonin gene-related peptide, substance $\mathrm{P}$ and protein kinase $\mathrm{C}$ in cultured dorsal root ganglion neurons following chronic exposure to $\mathrm{mu}$, delta and kappa opiates. Neuroscience 115, 441-453 (2002).

32. Munksgaard, S. B., Bendtsen, L. \& Jensen, R. H. Modulation of central sensitisation by detoxification in $\mathrm{MOH}$ : results of a 12-month detoxification study. Cephalalgia 33, 444-453 (2013).

33. Calabresi, P. \& Cupini, L. M. Medication-overuse headache: similarities with drug addiction. Trends Pharm. Sci. 26(Feb), 62-68 (2005).

34. Cupini, L. M., Sarchielli, P. \& Calabresi, P. Medication overuse headache: neurobiological, behavioural and therapeutic aspects. Pain 150, 222-224 (2010).

35. Jansen-Olesen, I., Tfelt-Hansen, P. \& Olesen, J. Animal migraine models for drug development: status and future perspectives. CNS Drugs 27, 1049-1068 (2013).

36. Olesen, J. The role of nitric oxide (NO) in migraine, tension-type headache and cluster headache. Pharm. Ther. 120, 157-171 (2008).

37. Goadsby, P. J., Edvinsson, L. \& Ekman, R. Vasoactive peptide release in the extracerebral circulation of humans during migraine headache. Ann. Neurol. 28, 183-187 (1990)

38. Sarchielli, P., Alberti, A., Codini, M., Floridi, A. \& Gallai, V. Nitric oxide metabolites, prostaglandins and trigeminal vasoactive peptides in internal jugular vein blood during spontaneous migraine attacks. Cephalalgia 20, 907-918 (2000).
39. Juhasz, G. et al. NO-induced migraine attack: strong increase in plasma calcitonin gene-related peptide (CGRP) concentration and negative correlation with platelet serotonin release. Pain 106, 461-470 (2003).

40. Green, A. L. et al. Increased susceptibility to cortical spreading depression in an animal model of medication-overuse headache. Cephalalgia 34, 594-604 (2013).

41. Ma, Q. P., Hill, R. \& Sirinathsinghji, D. Colocalization of CGRP with 5-HT1B/ $1 \mathrm{D}$ receptors and substance $\mathrm{P}$ in trigeminal ganglion neurons in rats. Eur. J. Neurosci. 13, 2099-2104 (2001).

42. Amin, F. M. et al. Magnetic resonance angiography of intracranial and extracranial arteries in patients with spontaneous migraine without aura: a cross-sectional study. Lancet Neurol. 12, 454-461 (2013).

43. Woolf, C. J. Central sensitization: implications for the diagnosis and treatment of pain. Pain 152, S2-S15 (2011).

44. Forsythe, P. \& Bienenstock, J. The mast cell-nerve functional unit: a key component of physiologic and pathophysiologic responses. Chem. Immunol. Allergy 98, 196-221 (2012).

45. Krabbe, A. A. \& Olesen, J. Headache provocation by continuous intravenous infusion of histamine. Clinical results and receptor mechanisms. Pain 8, 253-259 (1980).

46. Antonova, M., Wienecke, T., Olesen, J. \& Ashina, M. Prostaglandin E(2) induces immediate migraine-like attack in migraine patients without aura. Cephalalgia 32, 822-833 (2012).

47. Kushnir-Sukhov, N. M. et al. 5-Hydroxytryptamine induces mast cell adhesion and migration. J. Immunol. 177, 6422-6432 (2006).

\section{Acknowledgements}

This work is supported by grants from the CNRS and the Fondation pour la Recherche Médicale (FRM 2013 DEQ20130326482 to P.D.). We thank M. Mekaouche, A. Fernandez and Dr J. Wang (INSERM U1051) for technical assistance, and F. Maingret for supervising C.B. during her Master. We thank M. Masse for the gift of the CD31 antibody. C.B. was supported by the French ministry and Fondation pour la Recherche Médicale.

\section{Author contributions}

C.B. carried out the behavioral, biochemical, and electrophysiological studies and analyzed the data. J.H. performed electrophysiology. N.O. performed immunostaining studies. V.P. performed qPCR and western blot. J.R. provided help in behavioral auditory tests. A.D. discussed the project. C.B. and P.D. wrote the paper. P.D. supervised the project.

\section{Additional information}

Supplementary Information accompanies this paper at https://doi.org/10.1038/s41467019-12197-3.

Competing interests: The authors declare no competing interests.

Reprints and permission information is available online at http://npg.nature.com/ reprintsandpermissions/

Peer review information Nature Communications thanks Ingo Kurth, and the other anonymous, reviewer(s) for their contribution to the peer review of this work. Peer reviewer reports are available.

Publisher's note Springer Nature remains neutral with regard to jurisdictional claims in published maps and institutional affiliations.

Open Access This article is licensed under a Creative Commons Attribution 4.0 International License, which permits use, sharing, adaptation, distribution and reproduction in any medium or format, as long as you give appropriate credit to the original author(s) and the source, provide a link to the Creative Commons license, and indicate if changes were made. The images or other third party material in this article are included in the article's Creative Commons license, unless indicated otherwise in a credit line to the material. If material is not included in the article's Creative Commons license and your intended use is not permitted by statutory regulation or exceeds the permitted use, you will need to obtain permission directly from the copyright holder. To view a copy of this license, visit http://creativecommons.org/ licenses/by/4.0/.

(C) The Author(s) 2019, corrected publication 2021 